\title{
A Paradigm-Shift in Aerospace Vehicle Design Synthesis and Technology Forecasting
}

\author{
Loveneesh Rana, ${ }^{1}$ Thomas McCall, ${ }^{2}$ James Haley, ${ }^{3}$ Lex Gonzalez, ${ }^{4}$ Amen Omoragbon, ${ }^{5}$ Amit Oza, ${ }^{6}$ and Bernd \\ Chudoba. ${ }^{7}$ \\ AVD Laboratory, UT Arlington Dept. of Mechanical and Aerospace Engineering, Arlington, Tx, 76019, USA
}

\begin{abstract}
Classically, aerospace design synthesis methodologies have been developed for a specified problem, with limited or no adaptability and expandability for application to a new problem not considered in the original development. This inability eventually limits the application of a conventional ASDS towards exploration of novel concepts. Thus, the institutions using their proprietary legacy ASDS systems become confined within the product and technology range which is imposed by their system's limitations. Such a scenario presents a grave situation as it hinders innovation and exploration at the conceptual stage, the design phase where design freedom is maximum. In response to this need, a new modular system is being developed, tested, and applied at the Aerospace Vehicle Design (AVD) Laboratory at the University of Texas at Arlington. The system, called as the Aerospace Vehicle Design Database Management System (AVD-DBMS, or simply the DBMS), provides a unique capability of developing custom-tailored sizing codes specific to the problem at hand. In this regard, the DBMS is not a synthesis system by itself. Rather, it is a system that creates custom problem-specific synthesis architectures tailor made to address the needs of the user. Such a capability represents a paradigm-shift in the way conceptual design has been traditionally implemented. This paper presents an overview of the research effort of six researchers over a span of eight years. The primary focus of this paper is to provide the conceptual solution logic, the development process and system specification of the DBMS. Additionally, four distinct casestudies are presented that demonstrate DBMS' superior capability to explore novel design concepts and provide the decision maker with informed and smart decision-making support.
\end{abstract}

\section{Nomenclature}

= Aerospace Vehicle Design

= Conceptual Design

$C D$

$=$ Lifting Reentry Vehicle

= Space Access Vehicle

TOGW= Take Off Gross Weight

\footnotetext{
${ }^{1}$ Post-Doc Researcher, Aerospace Engineering, AVD Laboratory, UT Arlington, AIAA Member.

${ }^{2}$ PhD Candidate, AVD Laboratory, UT Arlington, Student AIAA Member.

${ }^{3} \mathrm{PhD}$ Candidate, AVD Laboratory, UT Arlington.

${ }^{4} \mathrm{PhD}$, Aerospace Engineering, AVD Laboratory, UT Arlington.

${ }^{5} \mathrm{PhD}$, Aerospace Engineering, AVD Laboratory, UT Arlington.

${ }^{6} \mathrm{PhD}$, Aerospace Engineering, AVD Laboratory, UT Arlington.

${ }^{7}$ Associate Professor, Director AVD Laboratory, UT Arlington, AIAA Member. 


\section{Introduction}

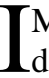
MAGINE the following scenario. A primary defense agency releases a request for proposal for the design and development of a non-conventional vehicle. It is a lucrative opportunity for the company and is a must win to remain viable and competitive. However, the company's toolsets are, although exceptional, tailor-made for the classical wingbody-empennage configuration and are not applicable to the non-traditional request. The company faces a costly situation. One, it can expend significant company resources to retool and train the design group (assuming it is even possible) or two, forgo the lucrative opportunity and subsequently decrease its competitive edge in the market.

Now consider, what if there were a tool that could be applied, which generically models any class of vehicles irrespective of the technology, mission, or hardware specified constraints? What could be the logical concept to develop such a tool? How could it be developed and how would it be applied? The objective of this paper is to present the details on such a capability.

The Conceptual Design (CD) phase is the most significant, least understood, and least innovated upon field in the aerospace industry. This is a primary reason why the aerospace industry has not been able to innovate and develop new flight vehicle concepts at the rate seen in the mid- $20^{\text {th }}$ century. Modern aircraft and rockets still look and operate on the same paradigms as they did in the 1960s.

It is crucial that the designer has a physics-based and parametrically driven design synthesis capability that supports informed decision-making and forecasting. Everything needs to be imagined, parametrically modeled or conceptualized before it can be invented. In this aspect, the CD phase represents the first point in the engineering frame of reference where the design engineer imagines a tangible product with physical understanding applied. As a point of creativity and design evaluation, the conceptual design engineer deserves and requires an approach and toolset that provides the capability to recognize the most viable and feasible design solutions in this early gestation phase of the program.

In response to this need, a new modular system is being developed, tested, and applied at the Aerospace Vehicle Design (AVD) Laboratory at the University of Texas at Arlington. The AVD Laboratory is a unique research environment that primarily focuses on advancing the state-of-the-art of conceptual design \& technology forecasting methodologies applied to atmospheric, space-launch and in-space vehicles. An Aerospace Systems Design Synthesis $\left(\mathrm{ASDS}^{8}\right.$ ) is a multi-disciplinary framework and the primary toolset used in the CD phase to establish concept feasibility and generate a solution space of potential design concepts for the specific mission requirements.

The system in discussion is the AVD Laboratory's Data-Base Management System or AVD-DBMS in short (also referred to as simply the DBMS). The DBMS provides a unique capability of developing custom-tailored sizing codes specific to the problem at hand. In this regard, the DBMS is not a synthesis system by itself. Rather, it is a system that creates problem-specific synthesis architectures tailor made to address the needs of the user. Such a capability represents a paradigm-shift in the way conceptual design has been traditionally implemented. Classically, synthesis systems have been developed for a specified problem with limited to no adaptability and expandability for application to a new problem not considered in the original development. In contrast, the DBMS provides a generic or flight vehicle configuration-independent framework that is designed to adapt to new problems and expand its capability when applied to new problems. The DBMS is designed from the outset to be 'flexible' and adaptable towards new product categories unlike the existing design synthesis tools that become more 'rigid' as product-category-specific aspects are hard-coded into their fundamental process over the life-span of the methodology.

The system was developed as a collective research effort between 2012-2016 by the AVD Laboratory researchers and co-authors Gonzalez, Omoragbon, and Oza. The prototype was first applied in a USAF research contract for a hypersonic technology demonstrator design study in 2015. Co-authors Rana, McCall and Haley have been and are advancing the system's capability through its application to multiple design exploration research initiatives for space access lifting reentry vehicles.

This document presents an overview of the research effort of six researchers over a span of eight years. The primary focus of this paper is: (1) to highlight the aerospace industry gap in the top-level planning and forecasting domain capability, (2) to introduce and explain the capability solution concept required to effectively mitigate this gap (AVD-

${ }^{8}$ ASDS is a representative term defined to refer commonly to a design synthesis methodology for aerospace vehicle systems. 
DBMS), and (3) explain the implementation of the solution concept as implemented in AVD-DBMS and provide proof of concept with past demonstration case-studies.

\section{Aerospace Systems Design Synthesis (ASDS) Review}

An ASDS is a systematic way to conceptually design the complex vehicle systems by considering the interdisciplinary integration among primary design disciplines. These disciplines are representative of the primary subsystems (geometry configuration, propulsion system, structures et al) that denote major functional characteristics of the vehicle (aerodynamics, propulsion, weight and volume respectively). Generally, a design synthesis methodology includes a set of analysis methods that solves for the main design disciplines, and a synthesis process logic that integrates the methods in a cohesive simulated environment, thus providing a conceptual design of a flight vehicle system and its primary subsystems. Figure 1 from a NASA technical memorandum report by Rowell and Korte [1] shows the launch vehicle conceptual design process and highlights how the design decisions propagate through the $\mathrm{CD}$ phase, thereby successively defining the technological and operational aspects of the vehicle. These decisions influence the final success or failure of the product and must be forecasted based on informed decision making at the early gestation phase. Clearly, such

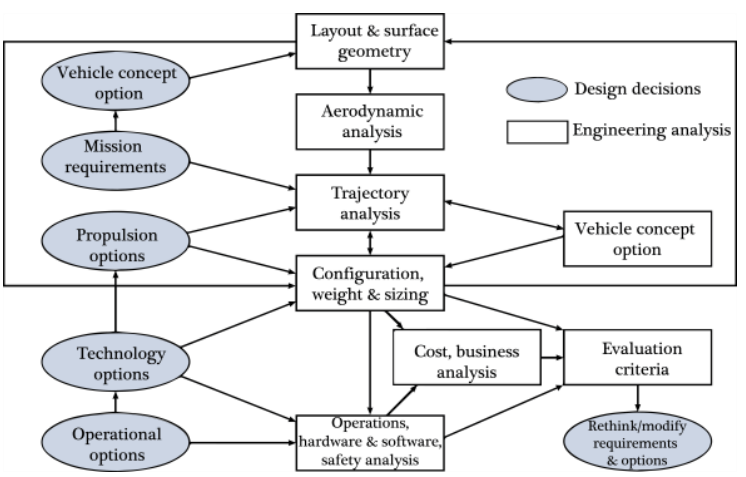

Figure 1 NASA Launch vehicle design process by Rowell and Korte [1]. capability is most desired by the chief decision maker, chief engineer and project engineers operating within a Future Projects Office type environment.

Through cumulative and continuing research efforts of several generations of researchers, the AVD laboratory has been expanding and developing an expansive library of valuable design data, design knowledge and synthesis processes, applicable to conceptual design synthesis. Several AVD Laboratory members (Chudoba [2], Huang [3], Coleman [4], Gonzalez [5], Omoragbon [6], Oza [7] et al) have studied and reviewed legacy and modern synthesis methodologies in great details throughout the aerospace product categories. The current research applies the lessons learned from these reviews conducted by AVD Laboratory members which entails the synopsis and key features of 126 unique synthesis systems, see Figure 2. To this effect, the next section discusses classical synthesis methodologies and it provides a modern synthesis system's overview and identifies the major drawbacks of existing

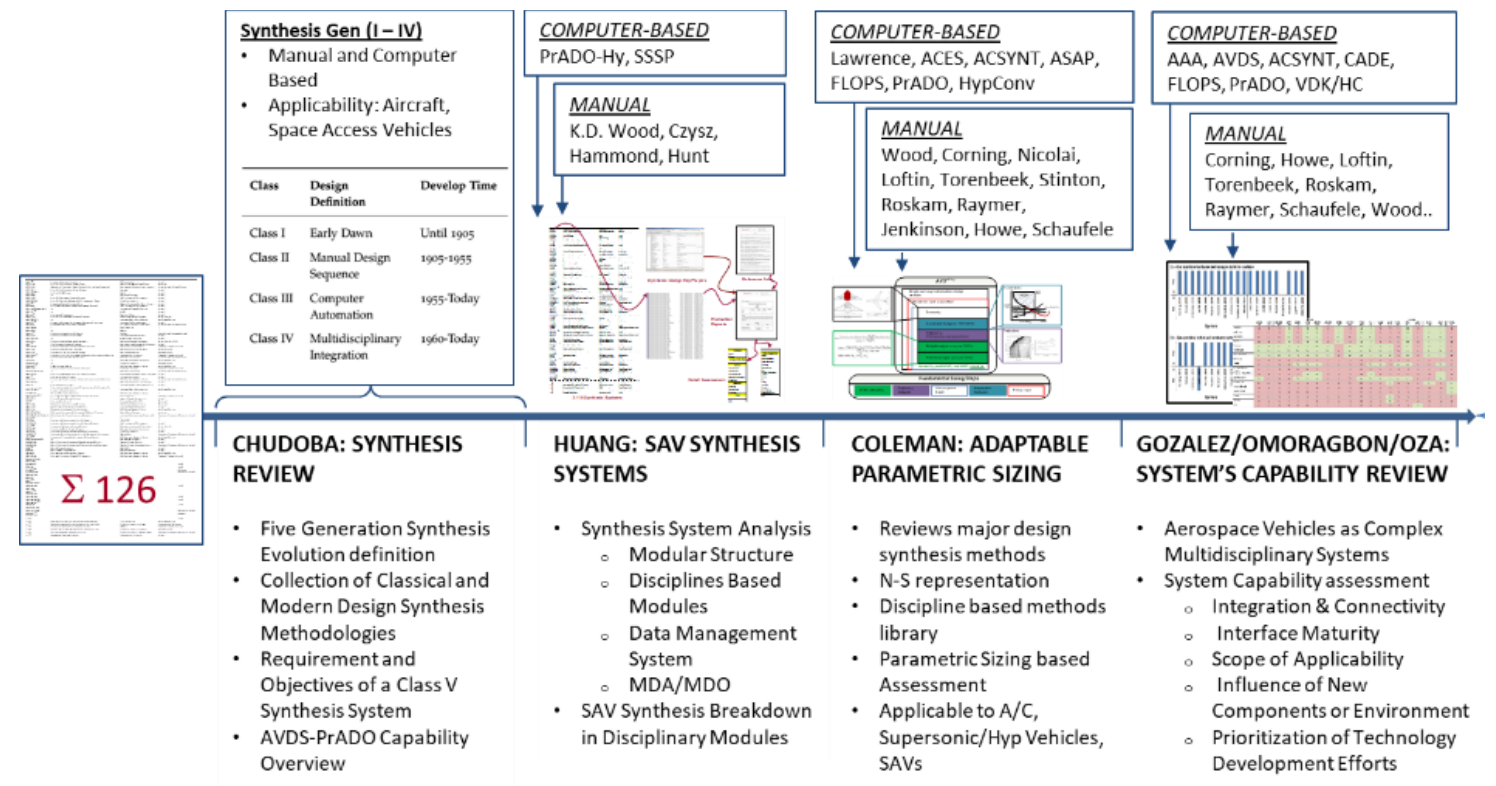

Figure 2 ASDS Synthesis System's Reviews by AVD. 
aerospace synthesis methodologies. This discussion defines the primary problem that is answered by the current study. Following the problem definition, the fundamental capability criteria requirements are defined for a bestpractice toolset to advance the status quo of the future advanced design and decision-making environment. These criteria act as the fundamental solution logic to the primary problem and are shown via selected case studies throughout the latter part of this document.

\section{A. Review of Existing Synthesis Capability - Problem Definition}

Chudoba [2] provides a historical review of the flight vehicle design synthesis systems and tracks the evolution in design methodologies from the legacy textbook synthesis processes to the modern-day computerized synthesis systems. A hierarchy of five generations of synthesis systems has been defined based on the level of increasing proficiency at integrating multi-disciplinary effects, see Table 1. The classification scheme selected distinguishes the multitude of vehicle analysis and synthesis approaches according to their modeling complexity, thereby expressing their limitations and potential. The first four generations of synthesis systems address chronologically the modelingcomplexity evolution of design approaches from 1905 to the present-day capability, highlighting primary characteristics of each class. This includes classical design approaches developed primarily towards aircraft sizing.

Table 1 Five generations of evolution of CD Synthesis approach by Chudoba [2]

\begin{tabular}{|c|c|c|c|}
\hline Class & $\begin{array}{l}\text { Design } \\
\text { Definition }\end{array}$ & Develop Time & Characteristics \\
\hline Class I & Early Dawn & Until 1905 & Trial and error approach, experiment, no systematic methodology \\
\hline Class II & $\begin{array}{l}\text { Manual Design } \\
\text { Sequence }\end{array}$ & $1905-1955$ & $\begin{array}{l}\text { Physical design transparency, parameter studies, standard } \\
\text { aircraft design handbooks }\end{array}$ \\
\hline Class III & $\begin{array}{l}\text { Computer } \\
\text { Automation }\end{array}$ & 1955-Today & $\begin{array}{l}\text { Reduced design cycles, detailed exploration of the design space, } \\
\text { discipline-specific software programs }\end{array}$ \\
\hline Class IV & $\begin{array}{l}\text { Multidisciplinary } \\
\text { Integration }\end{array}$ & 1960-Today & $\begin{array}{l}\text { Computerized design system, MDO, data sharing, centralized } \\
\text { design }\end{array}$ \\
\hline Class V & Generic Design & $\begin{array}{c}\text { Future } \\
\text { Generation }\end{array}$ & $\begin{array}{l}\text { Configuration independent, sophisticated design synthesis of a } \\
\text { user-defined aircraft, true inverse design capability, KBS }\end{array}$ \\
\hline
\end{tabular}

The first two classes of CD synthesis approaches were the first formalization of a multi-disciplinary framework in a standard textbook type methodologies. The transition from Class II to Class III represents the first use of computer automation in the design environment. These early design methodologies are found to focus on the selected disciplinespecific analysis but lack the multidisciplinary integration that is later implemented manually. Lovell comments, “... initial computer applications were confined to aspects of structural analysis and wing design. There was some resistance to the use of computers in initial project design because of the complex decision-making process involved. However, they enabled more detailed analyses to be made and hence allowed a greater range of carpet plots with additional overlays to be prepared to show the effects of configuration variables on performance. ..." [8]

Class IV synthesis systems are identified to involve multidisciplinary integration of a range of disciplinary analysis but are limited in application to single-point design optimization and mostly applicable to one specific vehicle configuration. The majority of synthesis systems up to Class IV are applicable only for subsonic and supersonic aircraft while only a selected few address the hypersonic vehicles class. Synthesis systems by Czysz (Hypersonic Convergence) [9] and Heinze (PrADO-Hy). [10] are identified as significant ASDS implementations of the Class IV type systems.

The assessment leads Chudoba to define the requirements for the next generation of Class V - Generic Synthesis Capability, which is identified as a design process rather than a design tool. In this regard, the focus here is on developing the capability over its application. The primary emphasis for this class is on the development of a dedicated modular disciplinary methods libraries and their integration into a central multi-disciplinary synthesis architecture.

In continuation of Chudoba's review of synthesis approaches, Huang [3], Coleman [4], Gonzalez [5], Omoragbon [6] and Oza [7] have conducted additional surveys of existing aerospace vehicle synthesis approaches. These reviews cover a total of 126 synthesis approaches which include legacy textbook design synthesis methodologies (e.g. Torenbeek [11], Roskam [12] etc.) and modern-day computerized synthesis systems (e.g. ASAP [13], FLOPS [14] etc.). 
Based on these reviews, the following conclusions provide an overview summary of the existing capability and major drawbacks of the traditional and current design methodologies (these methodologies fall under Class IV according to Chudoba's classification, see Table 1):

1. The majority of existing ASDS capabilities have been developed for the aircraft design application. Only selected few design synthesis systems exist that address hypersonic vehicle systems. Particularly, an efficient and dedicated design synthesis system for highly integrated hypersonic vehicles is still missing that could quantifiably forecast the mission-configuration-technology scenarios.

2. Synthesis is the primary integration capability that is the key to close (converge) the design through multidisciplinary parametric iterations. Very few synthesis systems can deliver a mathematical proof of design convergence while most past-to-present synthesis systems lack this crucial capability.

3. Another major drawback of current ASDS systems is that they are not able to efficiently generate the converged-design solution space topography and identify optimum design points. This capability requires that the synthesis system must have a rapid turn-around time and be able to converge on multiple design solution points.

4. Many design synthesis systems tend to have a common process-logic structure implemented via different computational procedures. Overall, the design methodologies of legacy synthesis systems tend not to be transparent. There is a lack of efficient computerized synthesis systems and multi-disciplinary interaction at the conceptual design level.

5. Existing synthesis systems have been developed specifically for a particular type of application (e.g. subsonic, supersonic, airbreather, rocket propulsion, wing body, lifting body etc.). This implies that many initial assumptions and methods are hard-coded at the development stages of the synthesis system, thus they are limited in application to only that specific use (a stovepipe situation, see Figure 3). As the system is used over time, it becomes stagnant and limited to the initial application boundaries. Clearly, there is no generic synthesis system for the flight vehicle conceptual design community that can produce a fair non-partial assessment or consistent comparison. This inability impedes the system's ability to assess all available design options and provide the best design solution independent of hardware, configuration and technology specifications.

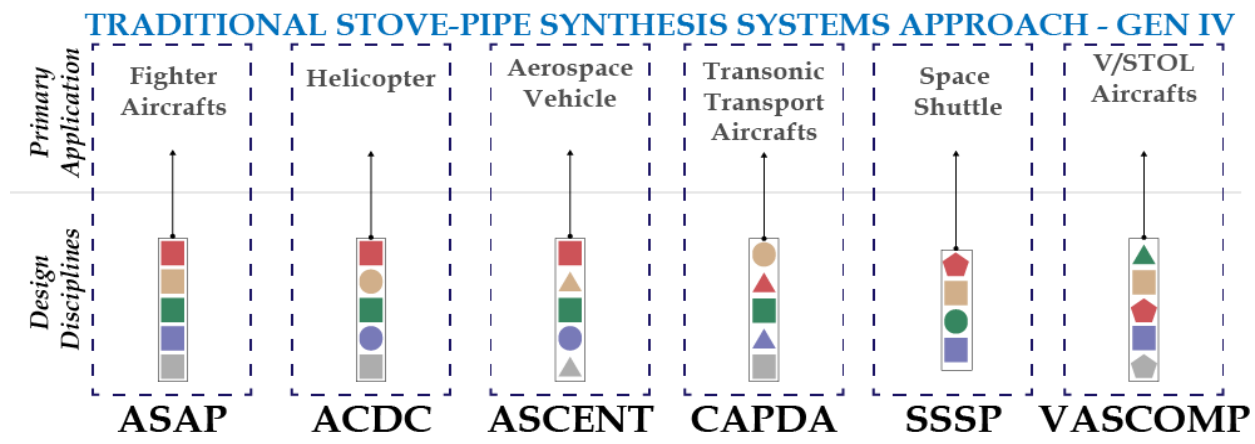

Figure 3 Examples of Existing Gen-IV ASDS Systems (designed for specific application).

\section{B. State-of-the-Art Design Synthesis System's Capability Requirements}

The previous section recognizes the major drawbacks of the existing synthesis capabilities based on the reviews of existing ASDS capabilities. Based on these drawbacks, this section identifies primary characteristic requirements of a best-practice synthesis capability that would act in the truest sense as a 'magic crystal ball forecasting tool'. The following discussion provides an overview synopsis of these characteristic features:

1. A Class V synthesis capability is distinctly identified as representative of the next generation forecasting system beyond the current state of the art Class IV systems. The primary aim while developing a Class V synthesis capability is the underlying development process, strategy and logic of the capability. Further, the Class V synthesis capability must be generic (configuration independent) in logic and be applicable to a wide array of flight vehicle configurations, rather than one specific class.

2. A state of the art Class V synthesis capability must be able to generate and screen a comprehensive design solution space for the $\mathrm{CD}$ phase, consisting of total-converged design concepts satisfying the required mission objectives. A design solution space is developed through the trades conducted for the primary design drivers that constitute the product being designed. Clearly, this requires that the Class V capability be able to execute trades for 
various configuration-technology-mission scenarios, and further define consistent comparative criteria to access the design solution for the technical and business-case feasibility.

3. In order to address the information-scarce nature of the CD phase, a Data-Base system is identified as a crucial requirement for the Class V synthesis systems. The Data-Base system is defined as a proficient toolbox to catalog and store the information specific to past-to-present projects, vehicles and primary design disciplines. This information aids the designer to make informed approximations and assumptions for the multi-disciplinary framework execution, and to define practical trade studies.

4. Parametric sizing is recognized as the vital system capability. Sizing is implemented in a mathematical framework to integrate the highest-of-importance disciplinary methods in a multi-disciplinary process and implement a convergence criterion to size the design concepts. In this aspect, distinct systems are required to manage a library of discipline-specific analysis methods and the multi-disciplinary integration \& convergence processes.

5. A Data-Base Management System (DBMS) is shown to be the crucial capability that enables a Class V synthesis system to model the widest variety of problems. This capability regulates the flow of information within the parametric sizing execution, controls data transfer between involved disciplinary modules and implements utilization of disciplinary methods in the multi-disciplinary framework. A robust data management system is found missing in legacy flight vehicle synthesis approaches and has been developed as $\mathrm{AVD}^{D B M S}$ to derive a prototype Class $\mathrm{V}$ synthesis capability.

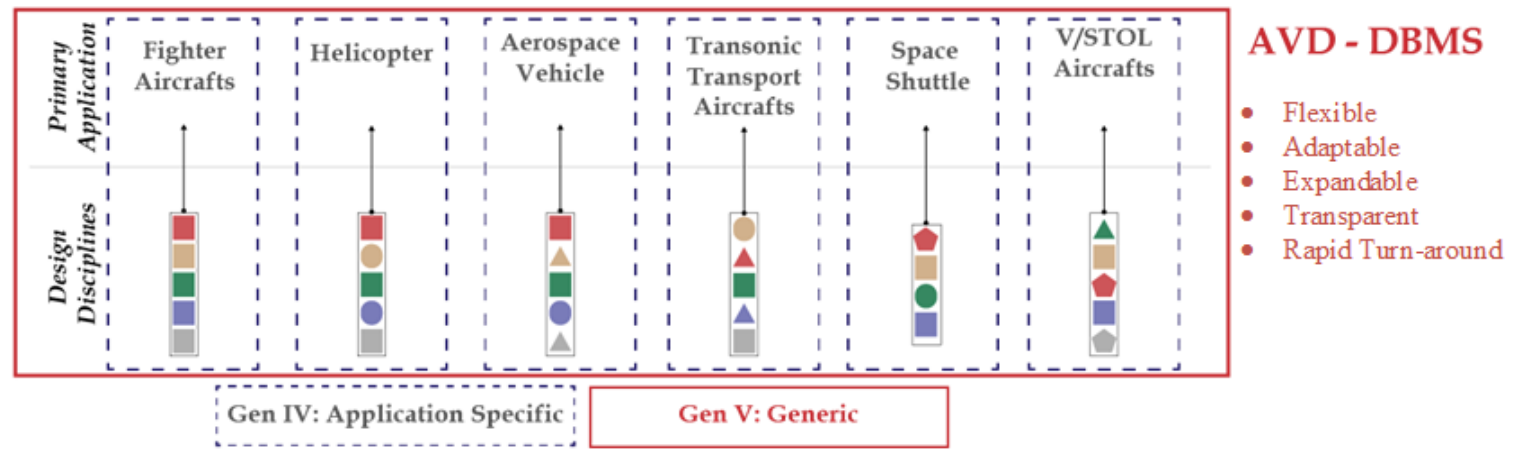

Figure 4 Generic Gen-V (AVD-DBMS) Capability Criteria Definition

These characteristic features thus form the fundamental characteristic requirements for a Gen- $\mathrm{V}$ generic design synthesis capability, the $\mathrm{AVD}^{D B M S}$. Further details regarding the system and its capabilities are described in the following chapters.

\section{Derived System Criteria}

Based on the synthesis system review, the resulting conclusion is that most computational toolsets are highlydeveloped high-inertia tools that generally require excessive source code familiarity and user time input to produce any significant modification to allow the system to address unique applications and configurations not originally considered in the tool development. As a result, necessity of a toolset is identified that is capable of rapid turnaround with a modular structure to adapt to new concepts and configurations, thus providing the designer with an advanced synthesis capability to develop a comprehensive solution space topography. Such a tool would require minimal user knowledge of the system, minimum user time investment for the output of a useable synthesis or sizing code with awareness of the developing world of artificial intelligence and potential future application.

The requirements of the system are determined by the now identified shortfalls of current synthesis systems and the necessities of the highly fluid and non-static nature of the conceptual design phase. The following are identified as the primary requirements criteria for a best-practice Class-V synthesis capability:

- Flexibility: modularity to handle any fidelity and unique concept/configuration;

- Adaptability: ability to adapt to the new problems, vehicles, configurations while maintaining the underlying structural framework;

- Expandability: ability to expand the underlying framework when additional new information is added;

- Transparency: transparent in terms of systems underlying mathematical framework, data flow, disciplinary inter-connections, full operation of process and system;

- Rapidity: quick turnaround, able to adapt and keep up with a rapid environment and quick turnaround deliverable times through various iterations. 


\section{Synthesis Tool Solution Concept and Development}

Having identified relevant literature, its applicability and capability, and having identified key features desirable in a Gen-V synthesis platform, a solution concept that integrates these insights is proposed in the first subsection. Following the conceptual summary, the second subsection provides details of the DBMS platform developed to implement the solution concept.

\section{A. General Solution Concept}

\section{Overall Methodology}

The DBMS concept is developed based on the principles of a Complex Multi-Disciplinary System (CMDS). Coleman [4] shows that aerospace synthesis systems are comprised of disciplinary methods as well as an analytic process. The disciplinary methods serve as the analysis modules of the system, whereas the analytic process as the system blueprint that controls the order and integration of the analysis modules. Coleman further explains that the choice of disciplinary methods is a function of the aircraft configuration, design mission and operational constraints defined for the problem. Accordingly, a CMDS is comprised of three classes of information: a description of the product being modelled, a definition of the analytic process being used to order and integrate the model, and a permutation of disciplinary methods performing the analysis of the model.

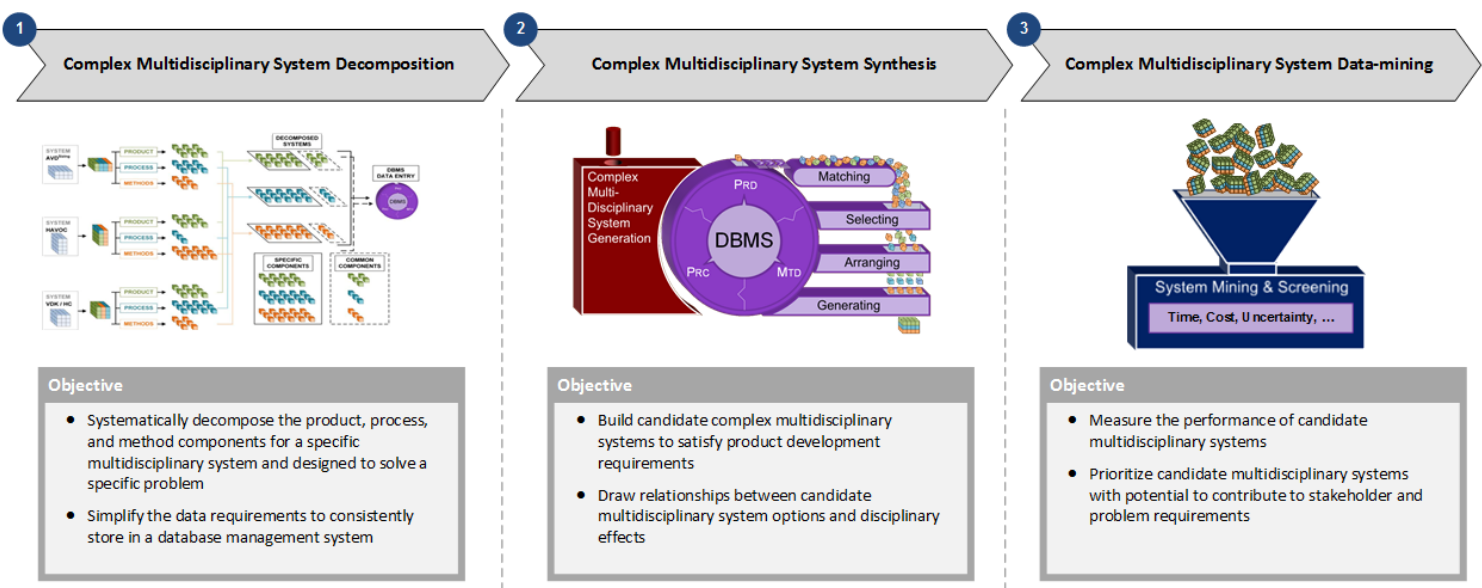

Figure 5 Conceptual methodology for developing the DBMS framework.

Figure 5 shows an overview of the primary steps executed to define the $\mathrm{AVD}^{D B M S}$ framework. The first step is the decomposition of existing technologies and existing ASDS methodologies into the three generic CMDS building block elements (explained in following sub-section). This step identifies fundamental elements required to build an ASDS. The next step is the composition of these elements into a system models to create customized problem-specific synthesis systems. The final step is the exploration of the different system models for the best solutions to the problem.

A general analogy to an automated car manufacturing plant further clarifies the DBMS development concept where a robot assembles a car with pre-stored components stored in various shelves in a warehouse. As per the requirement of what kind of a car needs to be produced, the robot goes through various shelves in the warehouse to pick the components specific to the car-type and initiated final assembly. In context of the DBMS development concept, the car represents an ASDS, the car-components represents fundamental building-blocks of an ASDS (the three CMDS building-blocks), the shelves in the warehouse represents the generic sub-categories of each CMDS block and provide a conceptual framework to store individual elements in the DBMS, the warehouse and the assembly robot is analogous to the DBMS where CMDS building blocks are stored and assembled when building a new ASDS (car).

\section{CMDS Building Blocks}

The three primary components of a CMDS are discussed with the following. These three separate classes represent the information necessary to characterize a design synthesis system. The product block describes the physical

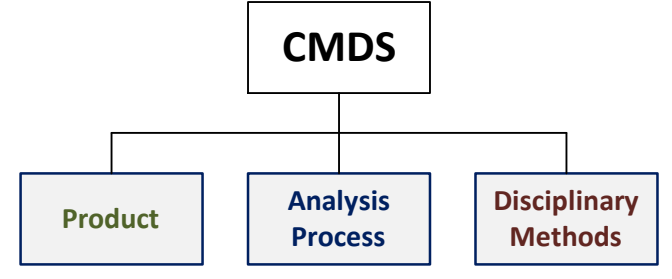

Figure 6 DBMS is developed following the principles of a Complex Multi-Disciplinary System (CMDS). 
characteristics of the artifact that is to be designed or acquired. The analysis process block prescribes the major steps to following evaluating the product. The disciplinary methods block describes the application of disciplinary principles or empirical data to obtain results for the different steps in the analysis process.

\section{Product}

The first component, the product refers to the physical representation of what is being modelled/solved. There are three considerations that describe the product: (1) what it does, (2) when it does it, and (3) the limitations or requirements for its operation. In context of an ASDS, it represents the flight vehicle and is comprised of three parts, namely; 1) Functional Subsystem, 2) Operational Events, and 3) Operational Requirements. Figure 7 shows an overview of the functional concept of the product component with each generic category and what it entails in relation to a flight vehicle. These three parts are defined as follows:

a) Functional Subsystem [individual hardware components added to achieve one or more primary functions];

b) Operational Event [operational attribute that is time dependent];

c) Operational Requirement [operational attribute that is time independent].

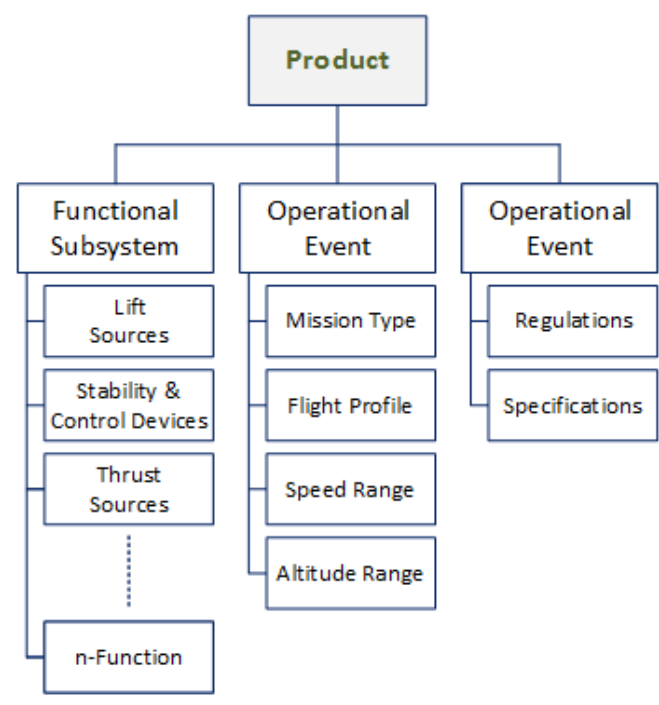

Figure 7 Conceptual buildup of the Primary Component-1: Product.

The breakdown clarifies that the product component of the CMDS

entails all aspects of the flight vehicle for which the ASDS is developed. It is common in classical ASDS methodologies to define vehicle hardware through the selection of disciplinary methods. An example of this can be seen in the PrADO [10], AVDS [4] and FLOPS [14] synthesis systems. In each case, the selection of methods in the input file defines the vehicle hardware being modelled. This places the responsibility entirely on the synthesis specialist to both: keep a listing of hardware inputs (separate from synthesis system), and to select disciplinary methods that represent that hardware (in the synthesis system). An outcome of this setting is the coupling of the definition of the vehicle hardware meant to be modelled with disciplinary analysis; the vehicle hardware is defined by the disciplinary methods selected. In order to decouple the definition of the vehicle hardware from the disciplinary analysis model, a hardware build-up methodology has been derived where the product is defined separately from the disciplinary methods.

\section{Analysis Process}

The second primary information class of the DBMS is the Analysis Process and is defined as any information relating to the overall organization and integration of an ASDS. Figure 8 shows the conceptual buildup of the Analysis Process. The Analysis Process is broken into two classes of information: System Elements, and Disciplinary Elements.

The System Elements block is composed of the System Process Variables that are the most important variables that controls and regulates the flow of the data in the MDA framework. They make up the objective function and are iterated until the objective function is satisfied, thus providing the mathematical convergence proof of the feasibility of the overall solution. The Disciplinary Variables compose the Disciplinary Elements and they are the primary top-level outputs of the involved disciplines. They are regulated by the Process Variables for each iteration.

\section{Disciplinary Method}

The third and final primary component of the DBMS is the Disciplinary Method. Figure 9 shows the conceptual buildup of this component into three main parts. This component primarily relates to the ability of the DBMS to handle and store individual disciplinary methods in the system. In order to consistently handle the data flow among the disciplines and to be able to

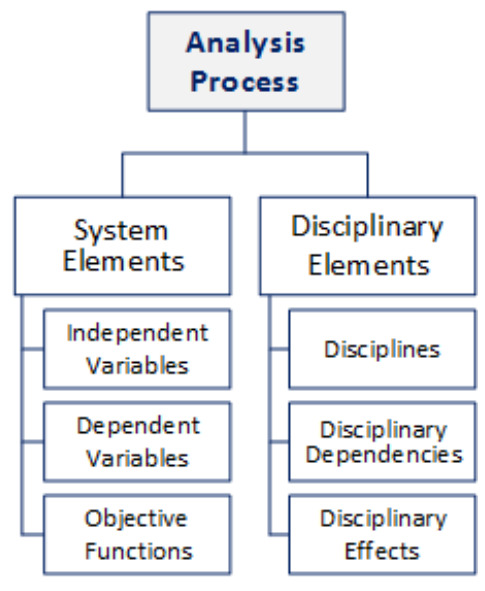

Figure 8 Conceptual buildup of the Primary Component-2: Analysis Process. 
combine the disciplines in the overarching MDA framework defined by the Analysis Process, the Disciplinary Method is associated to the product component identifying at which product node does the disciplinary method is applied. The Disciplinary Variables define the involved variables that the method is made up of and further specify the input and output variables for each method. The analysis (see Figure 9) part of the Disciplinary Method component contains the system of equations or the empirical data that connects input and output variables.

\section{CMDS Decomposition - System Development Concept}

The definition of the three primary CMDS components (Product, Analysis Process, and Disciplinary Methods) described thus far provides conceptual building blocks. Any past-to-present ASDS capability (classical text-book

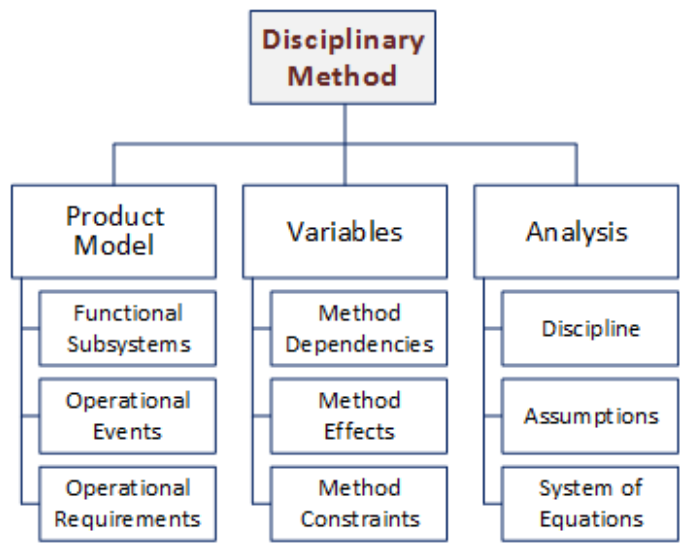

Figure 9 Conceptual buildup of the Primary Component-3: Disciplinary Methods. methodologies or modern synthesis systems) can be broken down into these three general elements. The first step in the DBMS development methodology is now to decompose the existing ASDS systems into these building blocks.

Complex Multidisciplinary Systems (CMDS) decomposition is done for defining the technology acquisition portfolio and evaluating the applicability of a conceptual design synthesis system to a design problem. Figure 10 shows the decomposition methodology for the aerospace CMDS (aka an ASDS) developed in this context. First, a given CMDS is decomposed into product, process and method blocks (as demonstrated by the AVD ${ }^{\text {Sizing, HAVOC, and }}$ VDK/HC blocks). Then these blocks are examined to identify common components that already exist in the database, followed by specific components that are unique to the CMDS. Finally, all the CMDS information is stored using AVD-DBMS. Using this methodology, the understanding of existing problems and capability to solve new problems increase as more CMDS are decomposed.

Another aspect of the CMDS decomposition process is the visibility of uniqueness of the parts that comprise a CMDS. The bottom right corner of Figure 10 shows that 'specific' and 'common' components are deciphered before entry into the DBMS. This prevents the duplication of building block data found in several CMDS. Additionally, the

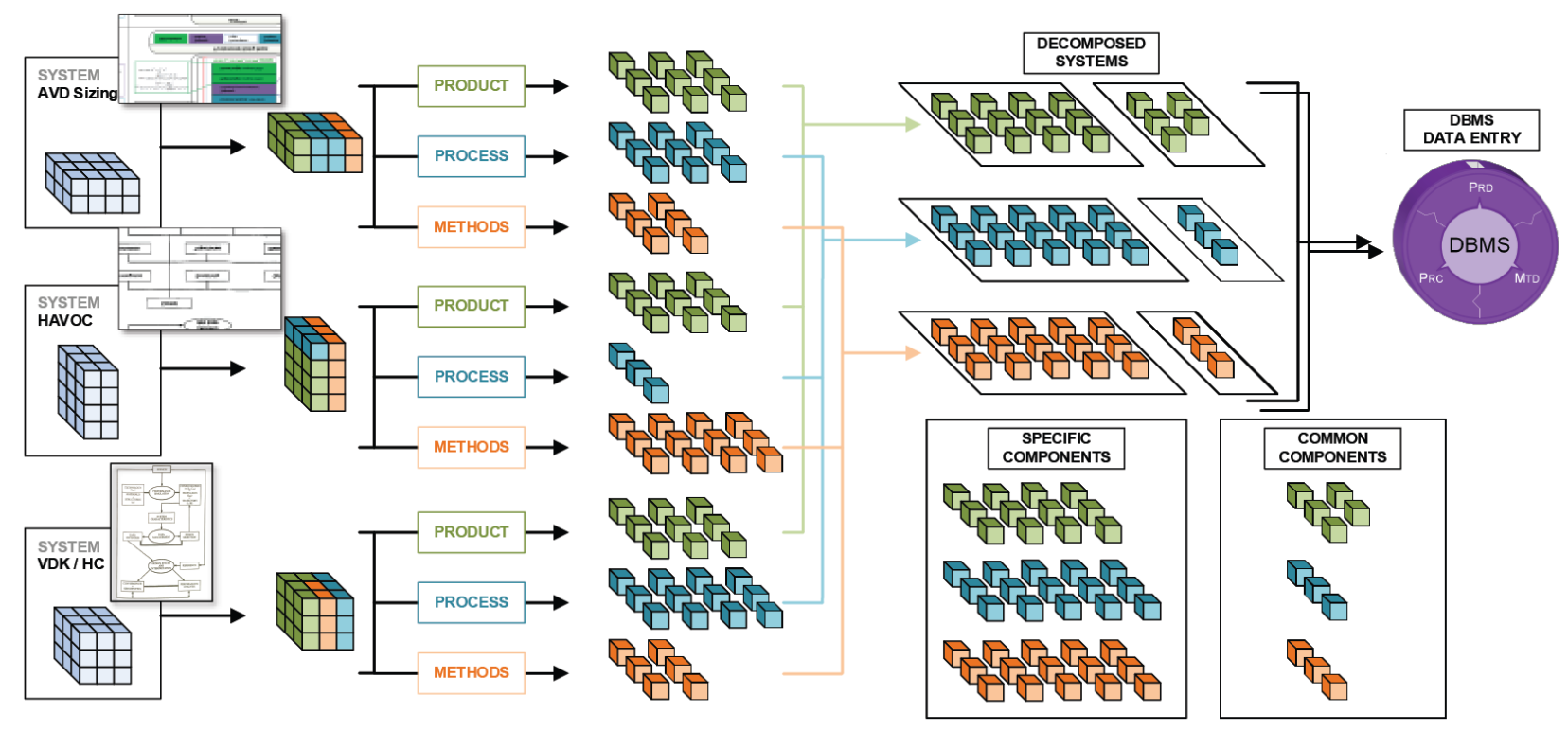

Figure 10 CMDS Decomposition Process. 
tracking of common building blocks allows the tracking of specific information as to how and where these components have been implemented in the past. The result of CMDS decomposition yields the building blocks for each of the parts of the synthesis system: Product, Analysis Process, and Disciplinary Methods. Each building block adheres to a standardized interface specific to the data type. This modular approach allows for building blocks of each type to be selected and integrated together to create new CMDS.

\section{CMDS Composition: Synthesis Development Execution}

The generation of a CMDS begins with the decision of a Product to be modeled and the Analysis Process used as the framework for analysis. The Product and Analysis Process breakdown results from a coupling of the stakeholder requirements and expected deliverables. This process is iterative, as the development lifecycle progresses and alters the scope of the problem. The Product and Analysis Process will need to be change accordingly. The strategy and/or

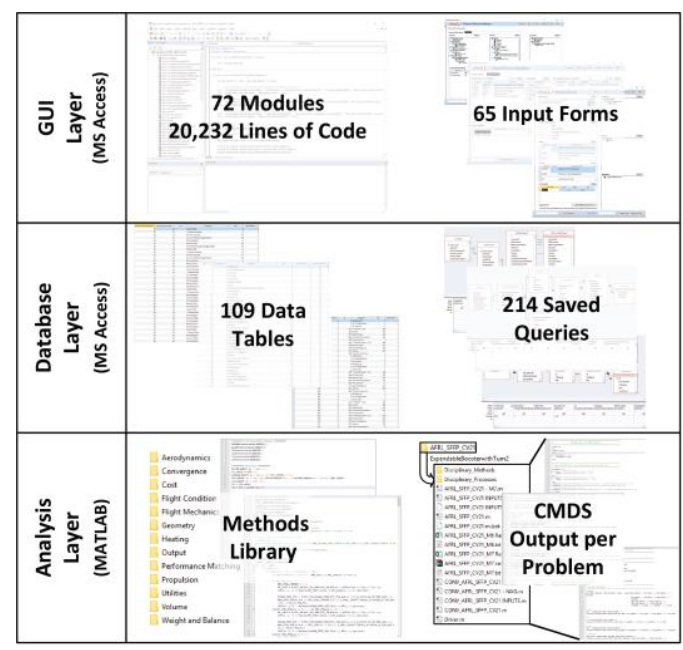

Figure 11 AVD-DBMS Three-Layer Architecture.

methodology used to create Product Breakdown and an Analysis Process as a function of Stakeholder Requirements is beyond the scope of the current research endeavor. As so, the CMDS Generation assumes that a Product and Analysis Process have been defined a priori. The methodology for the generation of a CMDS follows four sequential actionable steps: Matching, Selecting, Arranging and Generation. Each of these steps describe the action taken to the Disciplinary Method building blocks, in order to compose them with pre-defined Product and Analysis Process building blocks. It should be noted that the CMDS Generation Methodology assumes that a database management system exists with the capability to store the building block information from the decomposition process. The composition step is further described in greater detail in the following sub-section where the DBMS software implementation is explained next.

\section{B. AVD-DBMS: System Details}

Given the general solution description above, this section elaborates upon the implemented solution. The DBMS is not a design synthesis program in itself, rather, it is a software that creates unique, that means problem-tailored ASDS sizing codes. The process is executed in Microsoft ACCESS where the technical reference library, methods library, historical vehicle database, and sizing process architecture database are managed with this system.

AVD-DBMS has three layers: (1) Graphical User Interface (GUI) layer, (2) database layer, and (3) analysis layer, see Figure 11. The GUI layer is the means by which the user interacts with AVD-DBMS. It is created using MS Access forms that initiate VBA commands that control the database. The database layer contains SQL commands which manage data transfer between the GUI and database. It is also used to generate custom CMDS synthesis tools. The analysis layer, in the MATLAB environment, is where the CMDS synthesis tools are executed.

\section{Fundamental Building Blocks}

The DBMS is developed by following the fundamental logic of a CMDS and the three primary functional information classes, namely; 1) Product, 2) Analysis Process, and 3) Disciplinary Methods. Each component is made of three types of the building block input mechanisms referred as the utility modules. These modules are the basic elements through which the user interacts with the system and

References Input Form

The Reference Input Form is the mechanism enabling the capture of data and knowledge from source material and preparing it for use in the CMDS composition process.

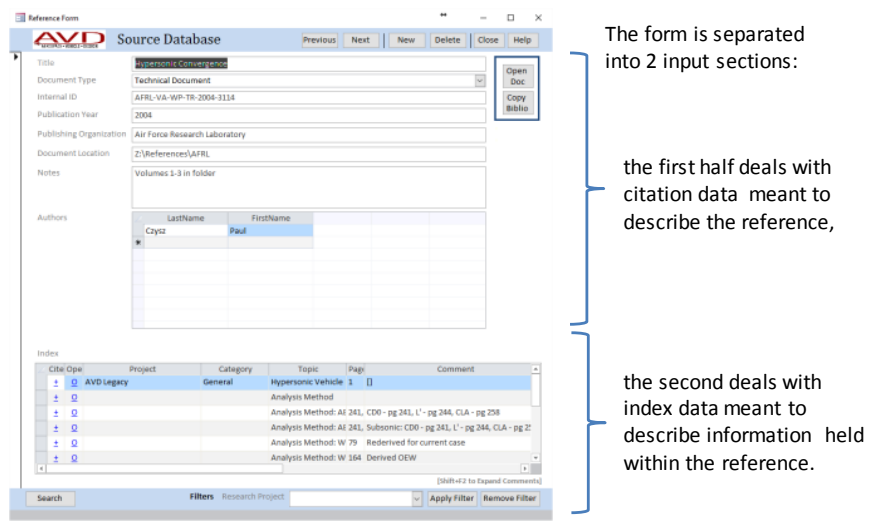

Figure 12 DBMS Software Building Block-1: Reference Input Form. 
are the building block to the DBMS software. The first building block, the Reference Input Forms is used to capture the data and knowledge from the reference source material. Figure 12 shows a reference input form from the DBMS (in MS Access) and identifies two input sections used to store and index the relevant information that is applied in the system.

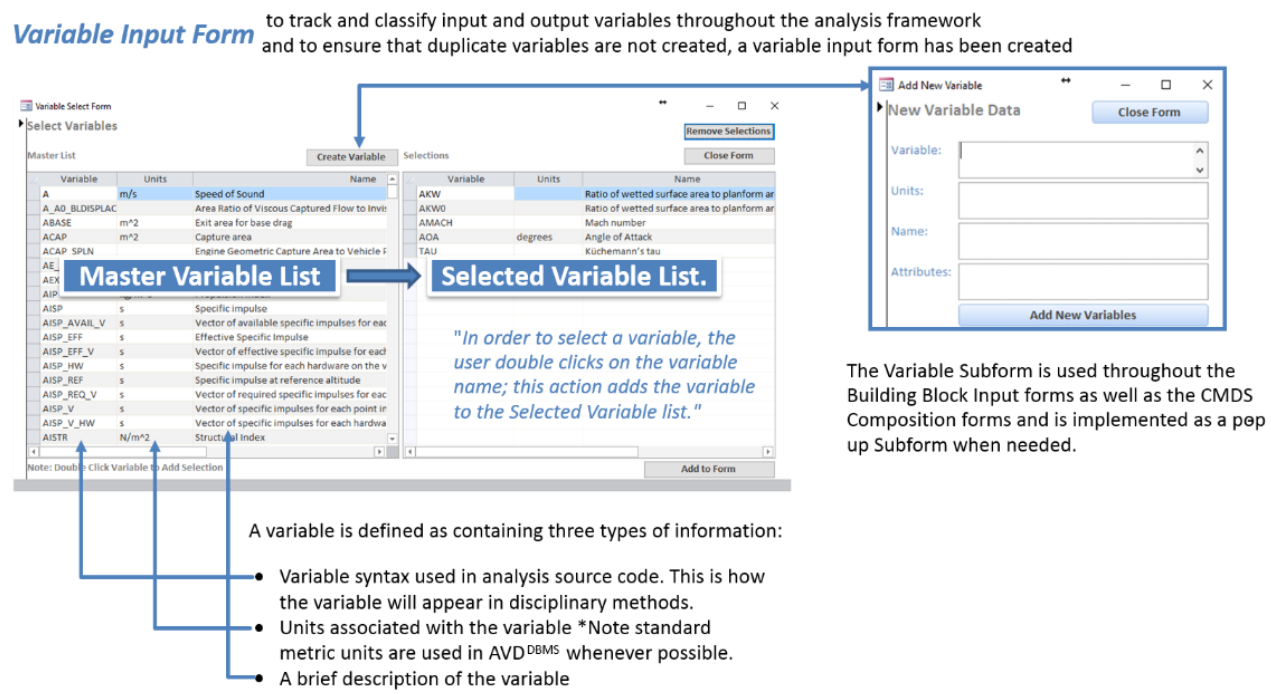

Figure 13 DBMS Software Building Block-2: Variable Input Form.

The second building block is the Variable Input Form that is used to store, track, select and classify input and output variables within the system. This mechanism ensures that duplicate variables are not created within the system and thus provides the ability to manage the variables throughout the analysis framework. Three specific information categories are assigned to each variable, (1) a Unique Syntax that shows how the variable appears in the system, (2) Units associated with each variable (SI unit system is followed), and (3) a brief description of the variable. Figure 13 shows the variable input form (as it appears in the system) with description of the main segments.

The third building block mechanism is the Input Tree Diagrams which provide a hierarchy structure to rapidly select various options and develop a vehicle, mission, operation or technology assessment. “... The Tree View control displays a hierarchical list of Node objects, each of which consists of a label and an optional bitmap. A Tree View is typically used to display the headings in a document, the entries in an index, the files and directories on a disk, or any other kind of information that might usefully be displayed as a hierarchy. ..." [15] Figure 14 shows the tree structure for its three main application in the system and describes the selecting mechanism to form a specific vehicle, mission or operation by selecting several nodes for each.

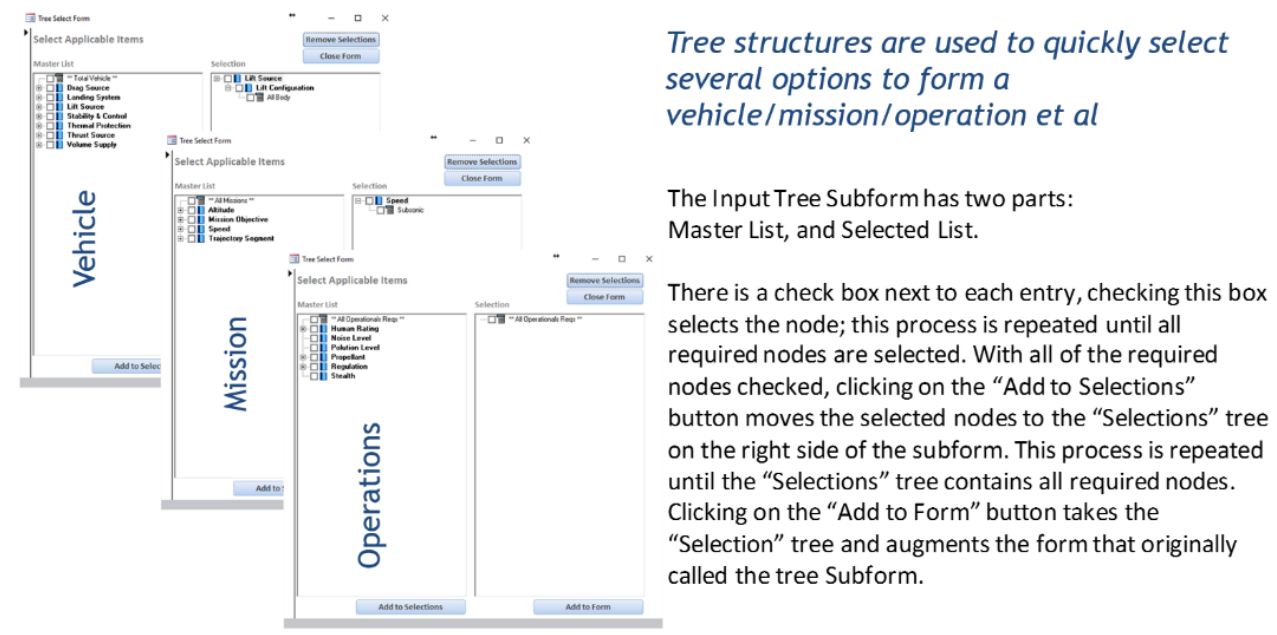

Figure 14 DBMS Software Building Block-3: Input Tree Structure 


\section{Execution of the DBMS to develop Customized ASDS Sizing Codes}

The DBMS system is assembled and managed in MS Access where it is executed in four sequential steps to finally create a custom flight vehicle sizing code. The conceptual logic of these four steps is described in the previous subsection as the CMDS composition logic. This section describes the execution of the four-steps composition process in the MS Access. The four executable steps are, 1) Matching, 2) Selecting, 3) Arranging, and 4) Generation, see Figure 16. All four steps are implemented in the DBMS through an Input Form Mechanism where the primary topcomponents, Product, Analysis Process, and Disciplinary Methods are combined in a step-wise manner. The sequential four steps make sure that the parametric connections and the data-structure is formulated correctly. At the end of the fourth step, Generation, the DBMS produces a sizing code architecture which is a custom tailored and stitched form of disciplinary method MATLAB files that are connected via the parametric connections defined in the DBMS.

The process of creating a synthesis sizing code begins by creating a new Product and Analysis Process that initiates a new ASDS architecture. The Product and the Analysis Process elements of the new architecture are developed prior to the execution of the four-step composition process using the input tree structure by selecting the problem specific components of each. Figure 15 shows an example snapshot of a Product and Analysis Process elements implemented in the MS Access. The first step is the Matching step where the vehicle's hardware, mission, and operations are specified by selecting a predefined product. Also, a part of the Matching step is the selection of the Analysis Process, which is selected from a predefined template.

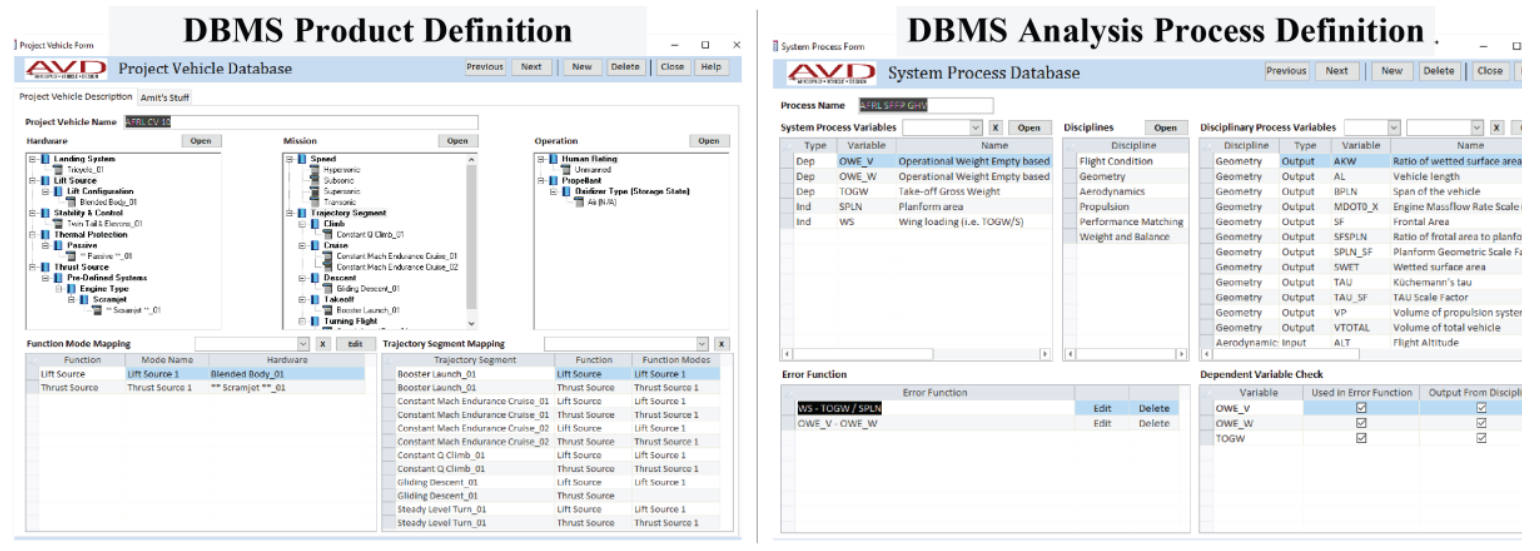

Figure 15 Product and Analysis Process examples in the DBMS MS Access platform.

After the selection of the predefined product and analysis process template, the next step, Selection, provides the user with a list of disciplinary methods which produce the output variables as required in the Analysis Process variables. This is a user dependent step where the user must make sure that the methods chosen are consistent with the vehicle and analysis process.

Once the methods are selected for each design discipline, the DBMS moves to the next step, Arranging, where the trajectory segments are asked to be placed in the sequence of mission profile. This sequence stitches the performance analysis methods in a sequence such that the total weight ratio and fuel fraction for the entire mission trajectory can be calculated. The Arranging step also makes sure that no two methods are being executed for the same flight phase for the same disciplines and thus provides user with options to specify the filter for how and where each method must be executed. This is specifically done for the Aerodynamic methods, where the methods specific to the Mach number range are assigned.

The Generation step is the final step of the process, where a variable based check is implemented for the entire product arrangement, disciplinary methods, and variables such that no inconsistent variable selection happens. Once this check is found to be satisfied, the DBMS has arranged all the disciplinary variables in a sequential order of execution following the logic defined in the Analysis Process for each discipline. At the end of this step, the system selects the MATLAB analysis files for each discipline that are stored on a server space and stitches them together in the variable execution logic defined in the MS Access system. Thus, the final MATLAB based flight vehicle sizing code is generated.

At this point, the user has a problem-specific custom-build sizing code in MATLAB files that does not require any inputs or interactions with the MS Access based DBMS. The user now starts to input the required disciplinary values 
as demanded by the disciplinary methods and project specification. The overall execution process is presented here in Figure 16 in the Nassi-Schnidermann (N-S) diagram format which shows specific instructions for each execution step.

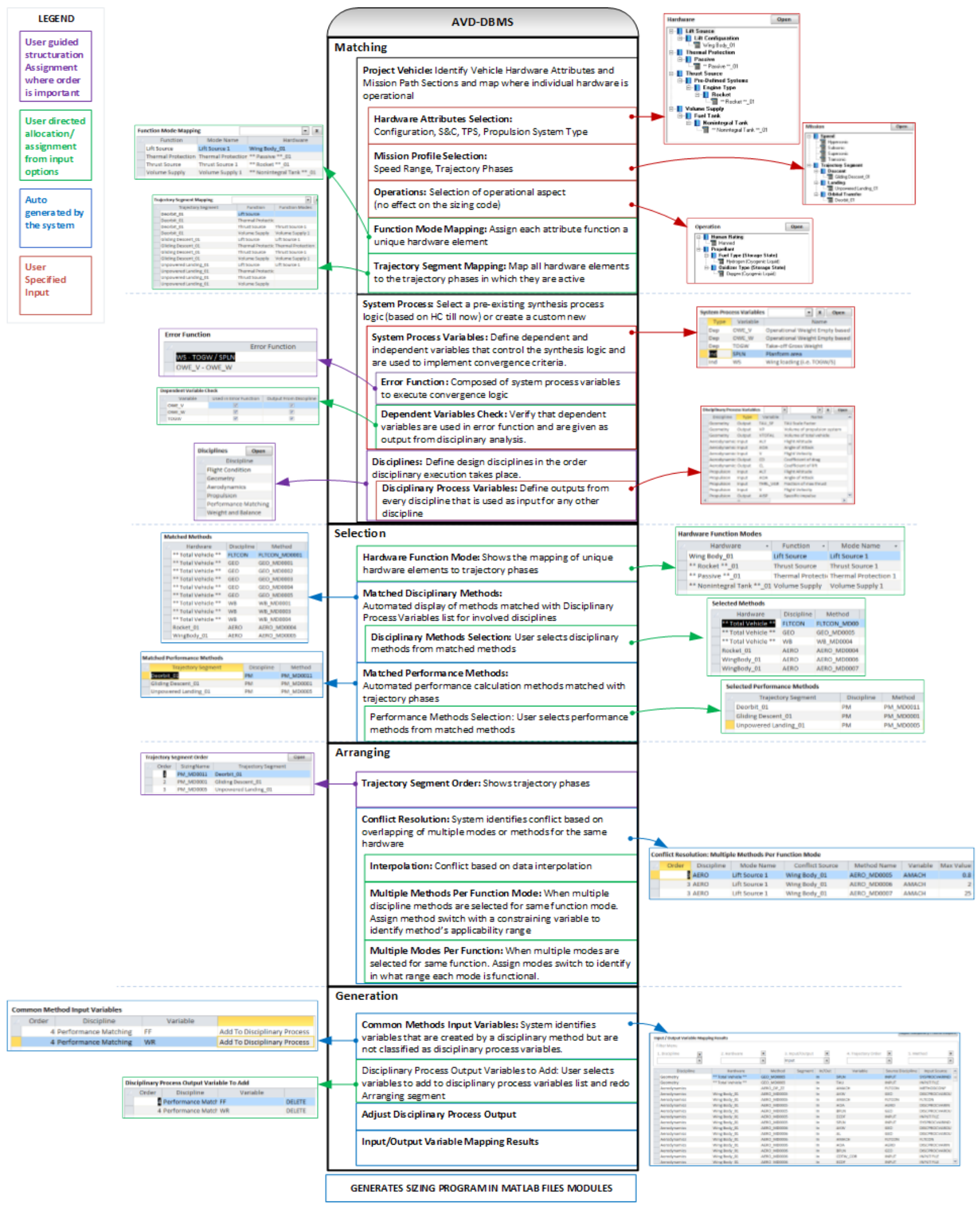

Figure 16 Execution process flow in the DBMS to create custom-built ASDS sizing codes. 


\section{Proof of Concept}

As previously mentioned, the DBMS is not a design synthesis program in itself, rather, it is a software that creates unique ASDS sizing codes specific to requirements of the problem. In this manner, the DBMS provides the designer with a higher-level control over the execution of different types of vehicles. This section provides an overview description of the four distinct case studies performed by applying the DBMS platform to create application-specific customized sizing codes. The first case-study entails sizing of a point-to-point hypersonic tech demonstrator vehicle on the lines of the X-24C scramjet vehicle. This case-study was executed by the co-authors; Gonzalez [5], Omoragbon [6], Oza [7], and Haley [16]. This was the first research study performed using the DBMS during its development phase and provided a proof-of-concept demonstration of the system's modular, adaptable, and expandable capabilities. With the execution of this case-study, the initial libraries for vehicle, technology, and disciplinary methods were populated for hypersonic flight vehicles.

While the first case-study focuses on atmospheric point-to-point hypersonic flight vehicles, the next three casestudies applied the DBMS towards Space Access Vehicles (SAV) design synthesis. These case-studies have been executed by co-authors; Rana [17], McCall, and Haley; and have been aimed at the development and exploration of a comprehensive design solution topography for the lifting reentry vehicles (LRVs) [18] for a LEO resupply mission. These case-studies provide a demonstration of the DBMS' superior capability in sizing vast amount of different tradestudies, since more than 1,400 distinct LRV concepts have been sized \& converged collectively in the last three casestudies over a span of 4 months [17]. These case-studies expanded the scope of the DBMS application by adding new disciplinary methods and creating specific product models within the DBMS framework. This execution also demonstrates the application of the DBMS to new vehicle categories by expanding the overall capability of the framework and provides a proof of its adaptable and expandable nature.

The general synthesis process for the case-studies is similar in terms of execution of the sizing code. The Küchermann slenderness parameter $\tau$ [19] [9] along with planform area defines and controls the overall geometric properties of the vehicle. For a single point design solution, planform area is iterated along with wing loading to converge the sizing process. The geometry module acts as the 'gearbox' of the synthesis system where the vehicle's geometry is defined in terms of vehicle slenderness parameter $(\tau)$ such that the geometry shape could be scaled efficiently using just $\tau$ as it accounts for weight, volume and aerodynamic effects collectively. Further iteration on $\tau$ and other mission-technology-configuration parameters populates a solution space consisting of individually converged total vehicle design points. The combination of mission-technology-configuration parameters defines the trade matrix and enables the designer to parametrically assess various distinct vehicle concepts options consistently and comprehensively. The overall design solution space continuum provides a carpet plot visual aid that is of significant value for the decision maker during the early design phase. The results presented here are represent the final solution space topographies containing numerous design points where each point is a fully converged vehicle. For further details on individual case-studies, the reader is referred to the specific references.

\section{A. Hypersonic Demonstrator}

\section{Case Study Description}

The DBMS has been first applied to demonstrate its unique capability as a part of the AFRL Summer Faculty Fellowship Program by Chudoba and Gozalez [20]. An AFRL initiative, the generic hypersonic vehicle (GHV) study was used as the verification case-study to validate the results of the DBMS. The GHV itself has been designed by the Air Force as an open source baseline research vehicle platform to enable easier collaboration with low security environments such as universities [21]. The DBMS results shows very good agreement with the GHV reference data. The overall system potential has been recognized by the USAF as the Fellowship Program extended to a research contract with AVD Services LLC., where the system has been further advanced and applied for the follow-on phases of the GHV study [20]. The DBMS development process, the GHV verification results and further details of the system are found in the PhD dissertations of co-authors and DBMS co-creators; Gonzalez [5], Omoragbon [6], and Oza [7].

Verifying the feasibility of the GHV has required sizing the configuration to similar mission requirements as it was designed for. According to Ref. [21] the mission requirements of the GHV are to cruise at Mach 6 with a dynamic pressure from 1,000 to 2,000 psf and maneuver at a maximum loading of $2 \mathrm{~g}$. On top of sizing the GHV for the Mach 6 cruise condition, the study expanded the GHV application to the Mach 7 condition to further investigate the design sensitivity to the overall mission constraints. 


\section{2. $\underline{\text { Results }}$}

Figure 17 presents the solution space result showing the effect of varying both tau and endurance cruise time on the vehicle planform area and TOGW. The pop-up on the top left of the solution space shows the results for a single point on the solution space. This is meant to emphasize the fact that each point on the solution space is a vehicle that has been converged to meet the input mission requirements; each point represents a closed (converged) solution in terms of weight and volume required as convergence criteria. The red lines on the solution space are the results of the design trade; solid lines represent line of constant $\tau$ whereas dashed line represent lines of constant endurance cruise time.

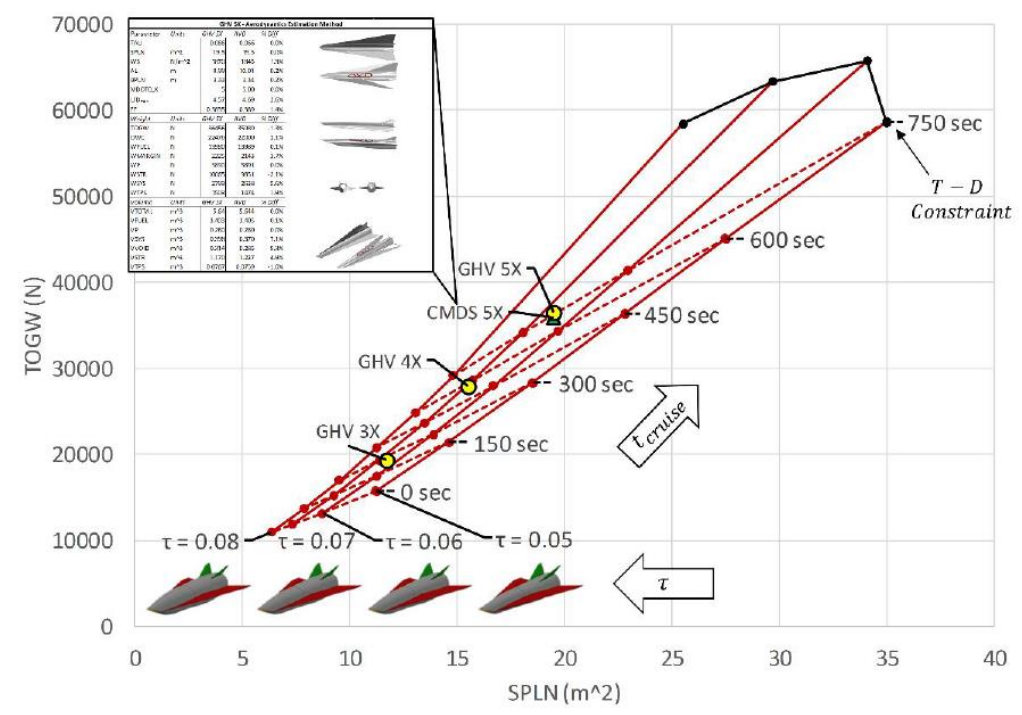

Figure 17 GHV solution space

A visual representation of the effect of $\tau$ on the GHV outer mold line can be seen along the bottom of the solution space. The black solid line represents the 'thrust minus drag' constraint line. For each value of tau, any increase in endurance cruise time creates a thrust requirement greater than the maximum capability of the vehicle. This constraint serves to cap our available solution space and provides a maximum for the design mission capability in terms of endurance cruise time.

Additional trade-studies have been performed including the booster stage for cruise conditions of Mach 6 and Mach 7 thus identifying extra constraints including the booster limitations. These results are shown below in Figure 18.

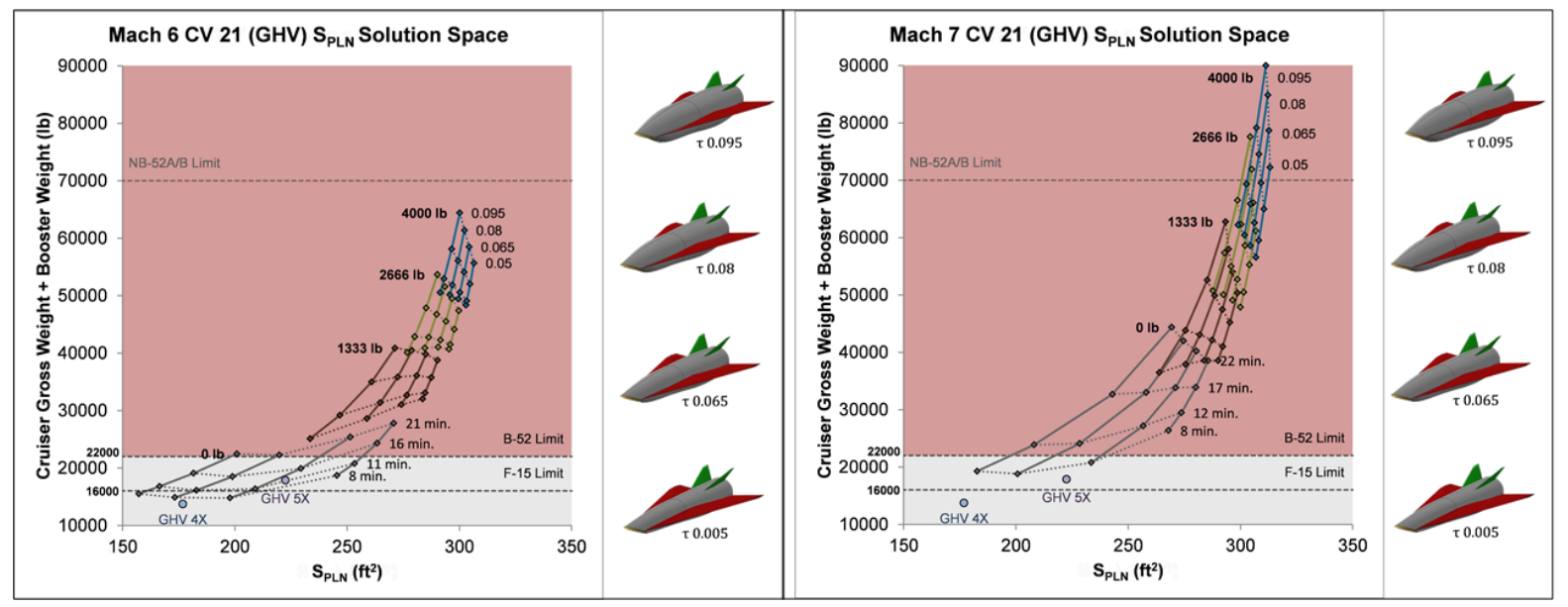

Figure 18 Mach 6 and Mach 7 Cruiser and Booster Solution Space 


\section{B. X-20 Dyna-Soar Reentry Vehicle Reverse Engineering}

\section{Case Study Description}

The second case-study has evaluated a legacy orbital reentry vehicle executing a reentry mission from Low Earth Orbit (LEO). The reference vehicle used is the 1957 concept, Boeing's X-20 Dyna-Soar which has been one of the first industry-scale initiatives to develop an operationally reusable orbital lifting reentry vehicle (hypersonic glider). The X-20 case-study has been the first application of the DBMS framework towards designing space access systems and it involved the creation of new databases, products, and disciplinary methods applicable for the space domain. Since the X-20 shows to have a data and knowledge rich history, this case-study acts as the verification and validation for the DBMS-generated SAV sizing codes and the results obtained. The case-study has been executed jointly by the co-authors Rana and McCall and has been documented in references [17] and [22].

\section{Results and Discussion}

Figure 19 shows the final solution space topography developed for the X-20 study by iterating on $\tau(0.18-0.26)$ and crew size, ANCREW (1 - 7). The solution space contains 63 design points where each point is a converged solution concept. Following the generation of the solution space topography, limiting constraints are applied based on the launch vehicle (TITAN III-C) payload limit and maximum heating limitation based on the TPS technology limit from the 1960s. Constraints implementation defines the feasible solution space where it is verified that the original X-20 design point lies comfortably within the feasible solution space. The reader is advised to consult references [17] and [22] for further details of this study.

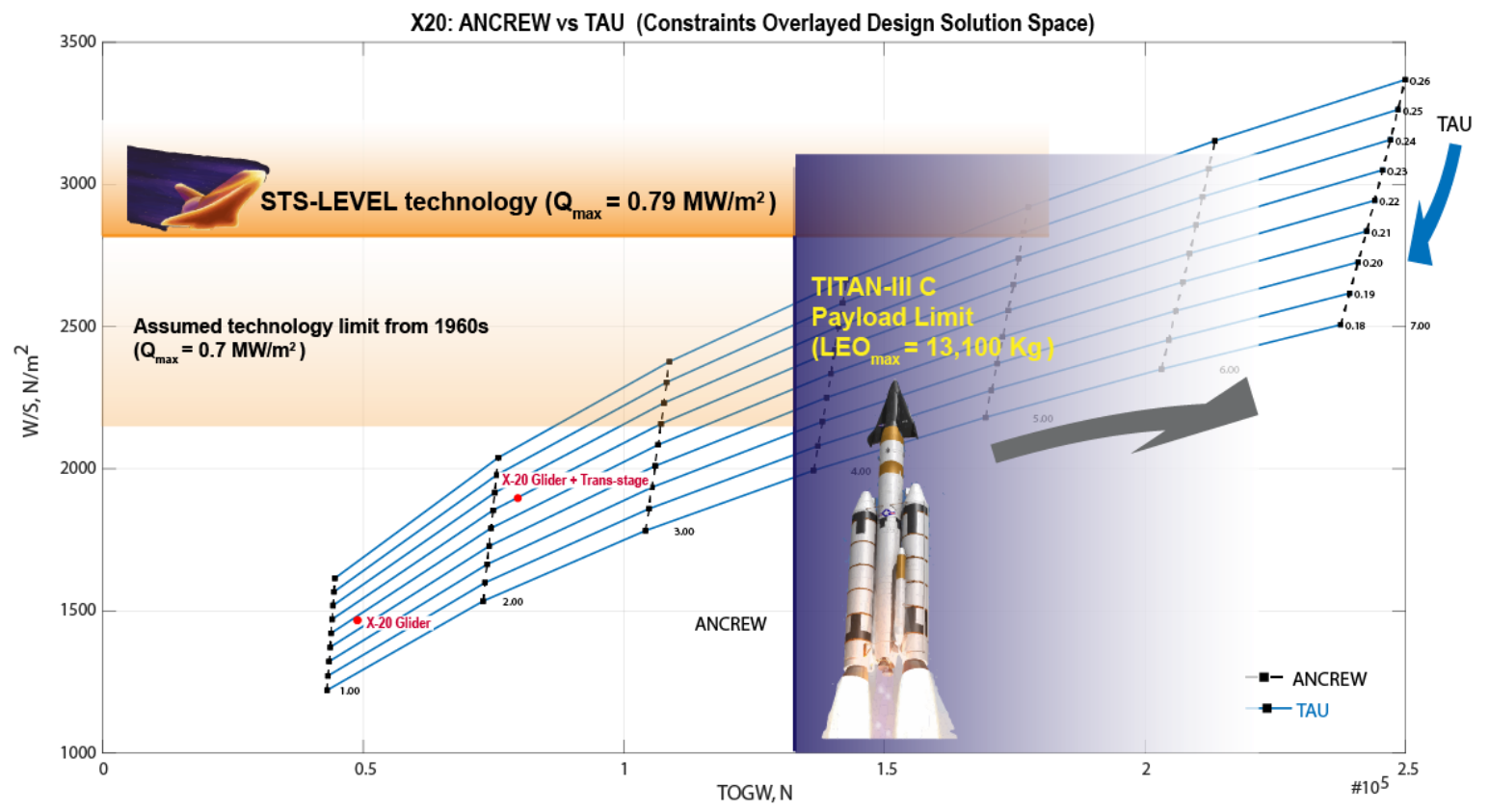

Figure 19 X-20 Case-Study Final Solution Space Topography.

\section{Generic Lifting-Body Reentry Vehicle Solution Space Exploration}

\section{Case Study Description}

This case-study has implemented the DBMS for a generic Lifting-Body (LB) configuration where multiple tradestudies have been executed on various mission, configuration, and technology parameters. The X-20 case-study addresses a legacy vehicle design which has a fixed geometry configuration with fixed mission objectives and technology requirements. Contrary to that, the generic LB case-study provides a wide range of design solutions which are addressed here. While the X-20 case-study has acted as a validation and verification case for the new product, data and disciplinary methods added in the DBMS for LRV application, the LB case-study has been aimed to build upon the added capability of the DBMS to explore the design solution space of the LRVs applying a generic LB configuration. Table 2 shows the overall trade-matrix where 8 distinct trade-studies (first column of the table) have 
Table 2 LB Case-Study Trade-Matrix

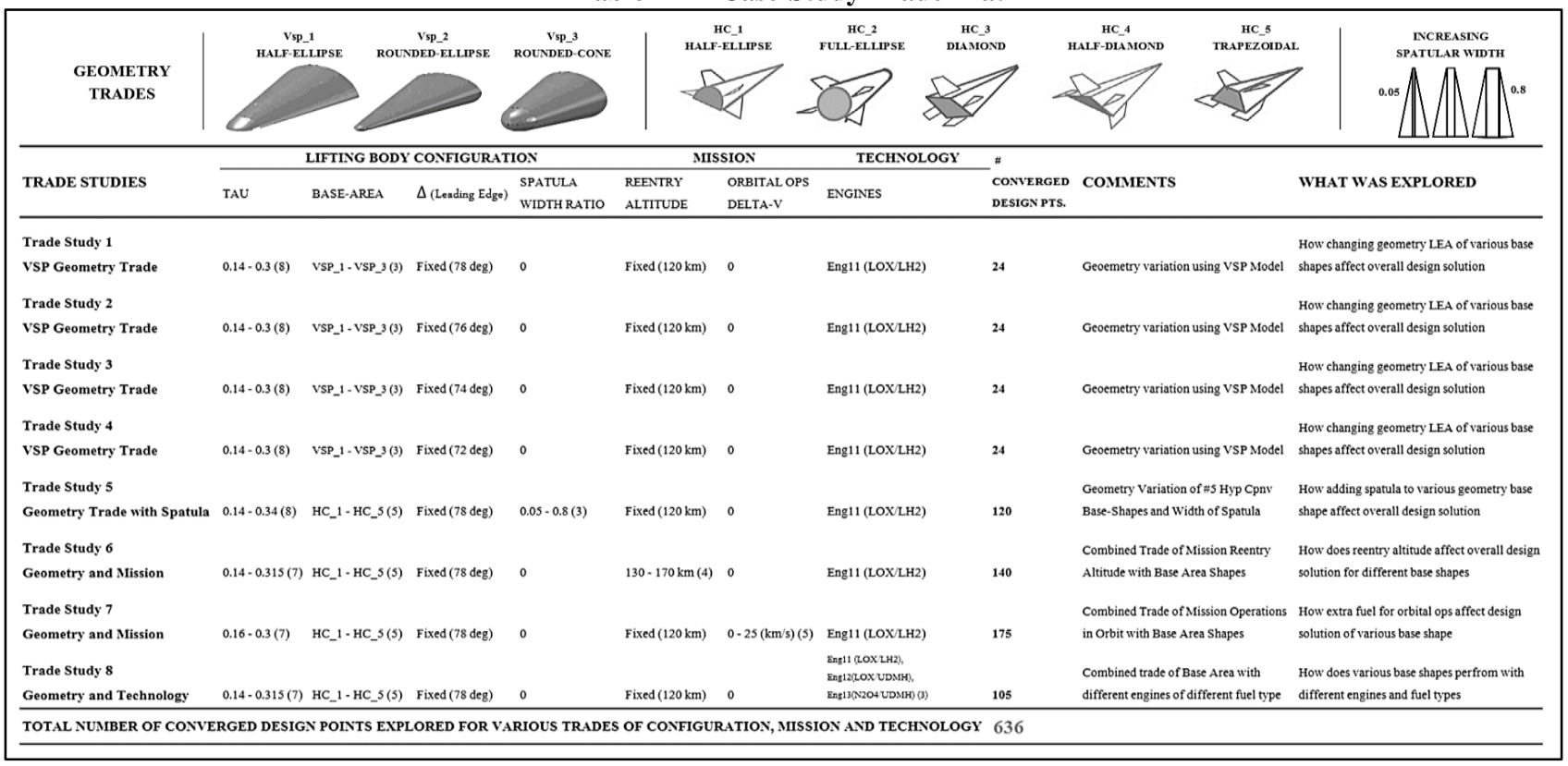

been defined through various configuration-mission-technology combinations. A new sizing code has been generated using the DBMS for each trade-study. As shown in the table, a $\tau$-sweep serves as the primary trade variable for volumetrically scaling the vehicle shape, while the other trade parameters are defined specifically based on the constraints of the disciplinary methods and execution capability and data-handling capacity of the software (MATLAB, OpenVSP etc.). The iteration variables are shown with their range while the number in the brackets denotes the number of steps of the iteration for the specific variable.

The study has generally covered a large range of trades that finally have resulted in an enormous solution space of 636 converged design concepts. It must be noted here that each design point has been sized in a similar manner as described in the single point design convergence of the X-20 case-study. That is, each design point has been iterated for planform and wing loading until the convergence criteria has been met. Clearly, every design point is a converged design concept that has calculated more than 200 internal and external disciplinary variables where each has around 30 disciplinary outputs.

\section{LB Holistic Measure}

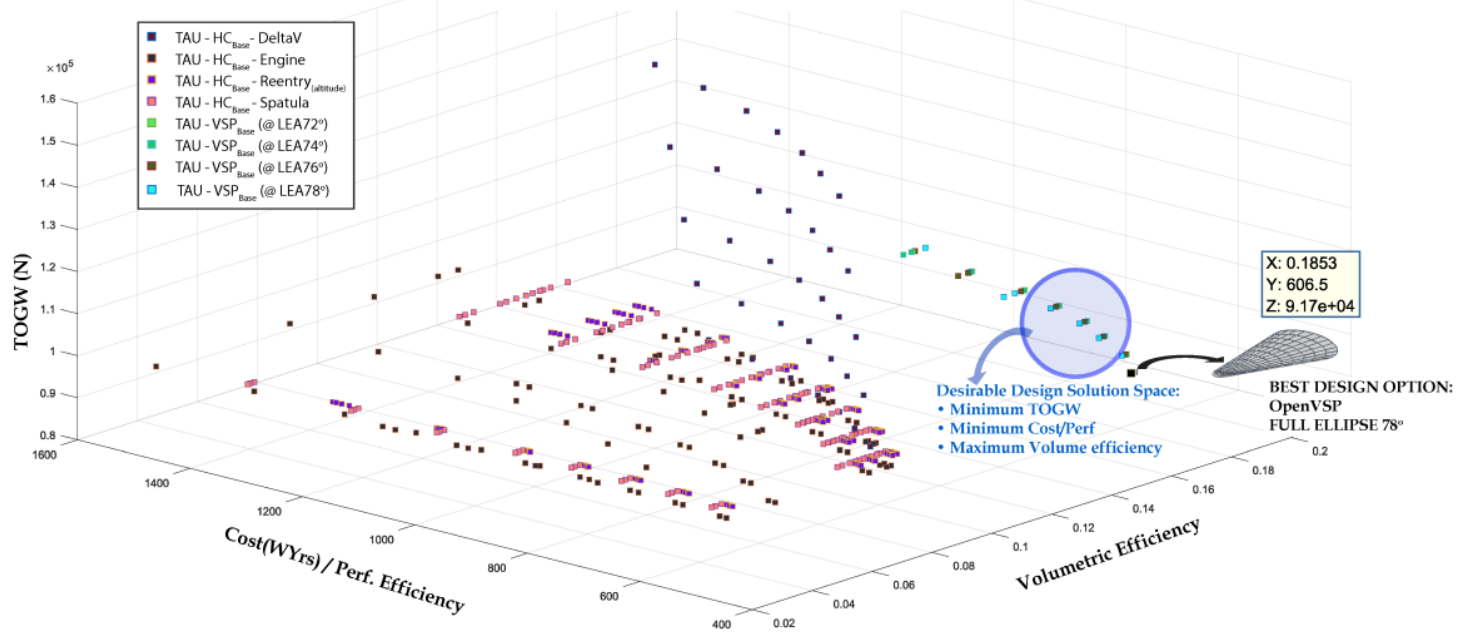

Figure 20 LB Case-Study Final Soluiton Space Topography (636 converged design points). 


\section{Results and Discussion}

The final results for all trade-studies are plotted together in one continuous solution space, see Figure 20. This solution space contains data for all 636 converged design solutions. The best design solution based on the three-axis visualization presented would be the one that has the least weight (TOGW), maximum volumetric efficiency and least cost/performance (note that Cost/Perf is the inverse of Perf/Cost which is same as miles-per-gallon or miles-per-dollar number for road vehicles). It can be seen that vehicles with a 78-degree leading edge angle and elliptical cross-section area shape are the most favorable on these measures. This case-study has been executed jointly by co-authors Rana, McCall, and Haley and has been documented in references [17] and [23].

\section{Generic Wing-Body Reentry Vehicle Solution Space Exploration}

\section{Case Study Description}

Similar to the generic LB case-study, an exploratory case has been executed for the generic wing-body (WB) configuration. As seen in Figure 21, three different WB geometry configuration profiles are created using the OpenVSP geometry modeling method. Different trade-studies have been executed in a similar manner as the LB case-study where each individual trade-study has been implemented by a unique sizing code generated by the DBMS. The geometry trades conducted are for vehicle configuration profile and leading-edge angle (LEA). A technology trade has been performed through the variation of fuel and oxidizer type.

Each trade study and the corresponding details are summarized in Table 3. Note that the range of $\tau$ and leading-edge angles are dependent on the constraints implemented by the geometry profile shape and OpenVSP model. The variation of the ranges is primarily due to the numerical limitation of the geometric solver in VSP analysis script. Although the maxima and minima of the $\tau$ and LEA are different, the number of steps for both in all geometric iterations are the same. The total number of design points sized for this study are 540 as shown in Table 3.

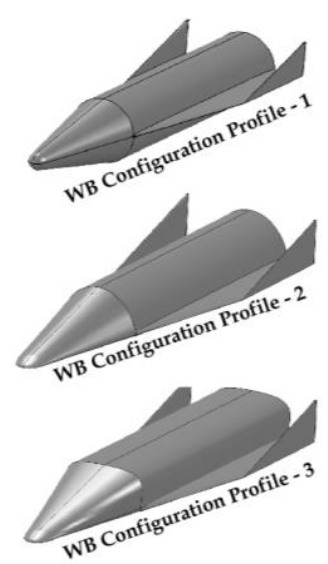

Figure 21 WB geometric profiles

Table 3 WB Case-Study Trade-Matrix

\begin{tabular}{|c|c|c|c|c|c|c|}
\hline & \multicolumn{3}{|c|}{ WING BODY CONFIGURATION } & \multirow{2}{*}{$\begin{array}{c}\text { MISSION } \\
\text { REENTRY } \\
\text { ALTITUDE } \\
\end{array}$} & \multirow{2}{*}{$\begin{array}{c}\text { TECHNOLOGY } \\
\text { ENGINES }\end{array}$} & \multirow{2}{*}{$\begin{array}{c}\# \\
\text { CONVERGED } \\
\text { DESIGN PTS. }\end{array}$} \\
\hline & TAU & Leading Edge Angle & $\begin{array}{l}\text { WB Configuration } \\
\text { Profile }\end{array}$ & & & \\
\hline $\begin{array}{l}\text { Trade Study } 1 \\
\text { VSP Geometry and Technology }\end{array}$ & $0.083-0.107(5)$ & Fixed(66 deg) & 1 & $140 \mathrm{~km}-95 \mathrm{~km}(4)$ & $\begin{array}{c}\text { Eng } 11 \text { (LO2/LH2), Eng } 12(\mathrm{LO} 2 / \mathrm{LH} 2) \\
\text { Eng } 13(\mathrm{~N} 2 \mathrm{O} 4 / \mathrm{UDMH})\end{array}$ & 60 \\
\hline $\begin{array}{l}\text { Trade Study } 2 \\
\text { VSP Geometry and TechnologyTrade }\end{array}$ & $0.084-0.104(5)$ & Fixed(68 deg) & 1 & $140 \mathrm{~km}-95 \mathrm{~km}(4)$ & $\begin{array}{c}\text { Eng } 11 \text { (LO2/LH2), Eng } 12 \text { (LO2/LH2), } \\
\text { Eng } 13(\mathrm{~N} 2 \mathrm{O} 4 / \mathrm{UDMH})\end{array}$ & 60 \\
\hline $\begin{array}{l}\text { Trade Study } 2 \\
\text { VSP Geometry and TechnologyTrade }\end{array}$ & $0.09-0.114(5)$ & Fixed(70 deg) & 1 & $140 \mathrm{~km}-95 \mathrm{~km}(4)$ & $\begin{array}{c}\text { Eng } 11 \text { (LO2/LH2), Eng } 12 \text { (LO2/LH2), } \\
\text { Eng } 13(\mathrm{~N} 2 \mathrm{O} 4 / \mathrm{UDMH})\end{array}$ & 60 \\
\hline $\begin{array}{l}\text { Trade Study } 4 \\
\text { VSP Geometry and TechnologyTrade }\end{array}$ & $0.11-0.178(5)$ & Fixed(72 deg) & 2 & $140 \mathrm{~km}-95 \mathrm{~km}(4)$ & $\begin{array}{c}\text { Eng } 11 \text { (LO2/LH2), Eng } 12(\mathrm{LO} 2 / \mathrm{LH} 2) \\
\text { Eng } 13(\mathrm{~N} 2 \mathrm{O} 4 / \mathrm{UDMH})\end{array}$ & 60 \\
\hline $\begin{array}{l}\text { Trade Study } 5 \\
\text { VSP Geometry and TechnologyTrade }\end{array}$ & $0.092-0.16(5)$ & Fixed(75 deg) & 2 & $140 \mathrm{~km}-95 \mathrm{~km}(4)$ & $\begin{array}{c}\text { Eng } 11 \text { (LO2/LH2), Eng } 12 \text { (LO2/LH2), } \\
\text { Eng } 13(\mathrm{~N} 2 \mathrm{O} 4 / \mathrm{UDMH})\end{array}$ & 60 \\
\hline $\begin{array}{l}\text { Trade Study } 6 \\
\text { VSP Geometry and TechnologyTrade }\end{array}$ & $0.09-0.13(5)$ & Fixed(78 deg) & 2 & $140 \mathrm{~km}-95 \mathrm{~km}(4)$ & $\begin{array}{c}\text { Eng } 11 \text { (LO2/LH2), Eng } 12 \text { (LO2/LH2), } \\
\text { Eng } 13(\mathrm{~N} 2 \mathrm{O} 4 / \mathrm{UDMH})\end{array}$ & 60 \\
\hline $\begin{array}{l}\text { Trade Study } 7 \\
\text { VSP Geometry and TechnologyTrade }\end{array}$ & $0.082-0.13(5)$ & Fixed(68 deg) & 3 & $140 \mathrm{~km}-95 \mathrm{~km}(4)$ & $\begin{array}{c}\text { Eng } 11 \text { (LO2/LH2), Eng } 12(\mathrm{LO} 2 / \mathrm{LH} 2) \\
\quad \text { Eng } 13(\mathrm{~N} 2 \mathrm{O} 4 / \mathrm{UDMH})\end{array}$ & 60 \\
\hline $\begin{array}{l}\text { Trade Study } 8 \\
\text { VSP Geometry and TechnologyTrade }\end{array}$ & $0.085-0.133(5)$ & Fixed(72 deg) & 3 & $140 \mathrm{~km}-95 \mathrm{~km}(4)$ & $\begin{array}{c}\text { Eng } 11 \text { (LO2/LH2), Eng } 12 \text { (LO2/LH2), } \\
\text { Eng } 13(\mathrm{~N} 2 \mathrm{O} 4 / \mathrm{UDMH})\end{array}$ & 60 \\
\hline $\begin{array}{l}\text { Trade Study } 9 \\
\text { VSP Geometry and TechnologyTrade }\end{array}$ & $0.082-0.11(5)$ & Fixed(76 deg) & 3 & $140 \mathrm{~km}-95 \mathrm{~km}(4)$ & $\begin{array}{c}\text { Eng } 11 \text { (LO2/LH2), Eng } 12(\mathrm{LO} 2 / \mathrm{LH} 2) \text {, } \\
\text { Eng } 13(\mathrm{~N} 2 \mathrm{O} 4 / \mathrm{UDMH})\end{array}$ & 60 \\
\hline TOTAL NUMBER OF CONVERGED D & ESIGN POIN? & TS EXPLORED & OR VARIO & DF CONFIGUI & N, MISSION, AND TECHNOL & 540 \\
\hline
\end{tabular}




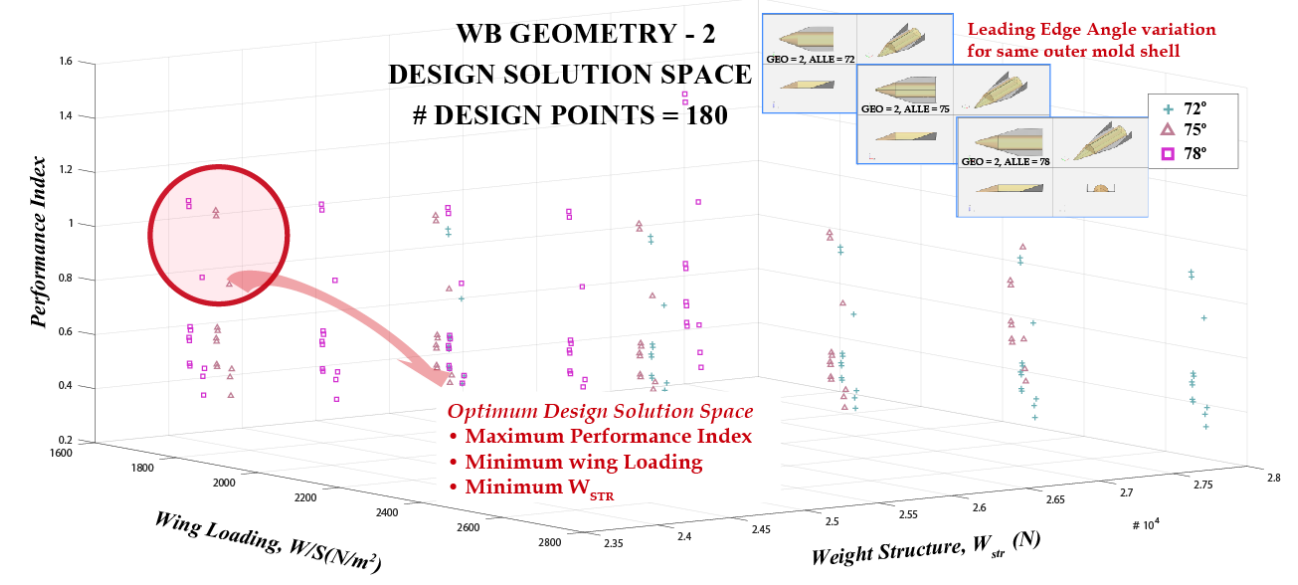

Figure 22 WB Geometry Profile-2 Soluiton Space Topography (180 converged design points).

\section{Results and Discussion}

Figure 22 first shows the example of a design solution continuum for the geometry profile- 2 with all trades of engines and reentry altitudes (Trade Studies 1, 2, 3 in Table 3). Examining the specific wing-body geometry solution space, there exist an optimal solution at the minimum wing loading, structural weight and highest performance index. This area is denoted by the red circle and is populated by both 78-degree and 75-degree leading edge angle vehicles. The highest performance is associated with the 78-degree leading edge although this solution space shows that the 75degree vehicles are not much further away and could be an alternative if further studies show an advantage for lower leading-edge angles.

The complete solution space continuum is presented in Figure 23 visualizing and parametrically comparing all 540 converged solutions in one single topography plot. The relationship between wing loading and structural weight is positive and seen increasing with a distinct difference between two sets of data which correspond to different engines using different fuel types (as seen on the left-side plot of Figure 23). Rotation of the solution space to show the performance index reveals (as seen on the right-side plot of Figure 23) that not all points along those lines are equal and there is a wide spread of performance related to engine $I_{\mathrm{SP}}, L / D$, and reentry velocity. The range of values for each vehicle shape are also different (denoted by color) which is attributed to a difference in feasible tau ranges for each shape. This is a feature of the geometry type and cannot be greatly influenced by modifying the geometry i.e. a cylinder has higher volumetric efficiency than a half cylinder. The WB case-study was executed jointly by co-authors Rana, McCall, and Haley and has been documented in references [17] and [24].

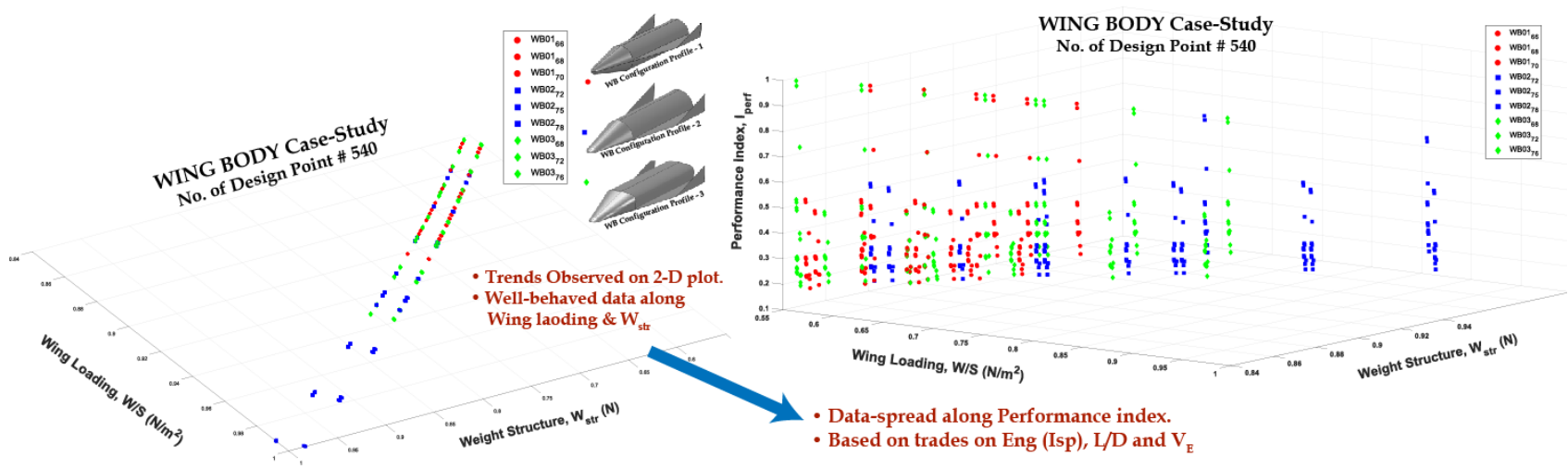

Figure 23 WB Case-Study Final Soluiton Space Topography (540 converged design points). 


\section{Conclusion}

The primary objective of this study was to identify and test a composable synthesis architecture toolset for aerospace system design synthesis. A thorough review of the existing ASDS capabilities has been conducted which illustrated the need for the next generation of the ASDS. As shown in Figure 24, current standard ASDS capability resides at the Gen-IV class.

The research investigation presented in this paper communicates the successful endeavor to develop a state-of-theart Gen-V capability, the AVD-DBMS. The research has outlined the defining system capability criteria. Following this, a solution concept logic has been described with the details of the DBMS software along with its execution process. Eventually, overview details are provided for four distinct research studies where the DBMS has been successfully applied and verified, thus demonstrating the superior capability of the system.

The DBMS has been shown as the current paradigm-shift capability in the ASDS evolution as it is paving the way to be a Gen-V platform that is capable of generating problem specified unique sizing codes (traditionally represented by Gen-IV ASDS). This capability is identified to be a significant advancement in synthesis capability. In order to demonstrate, the DBMS has been executed for the highly design-sensitive categories of hypersonic airbreather demonstrator and space access systems.

First two case studies serve to validate and verify the unique sizing codes generated by the DBMS. The next two generic (LB and WB) exploration case-studies provide a demonstration of the DBMS' modular, adaptable and expandable nature. The DBMS is shown to provide the flexibility to rapidly create a new sizing code specifically tailored for independent trade execution. This allows to parallel-execute multiple sizing studies, thus enabling designers to generate a vast number of converged solutions. Such a capability will allow designers to explore the complete design solution space and parametrically compare distinct design options consistently.

For future development, the generic synthesis tool generation concept will be translated into an artificial intelligence (AI) design environment that will serve as an assistant to the designer. Additionally, the system will be used to develop a machine learning based synthesis tool, to which the outputs of both systems will be compared. This capability is identified as the Gen-VI in ASDS evolution as shown in Figure 24. The AVD Laboratory is currently working towards augmenting the DBMS with an AI capability and is developing a true forecasting capability [25].

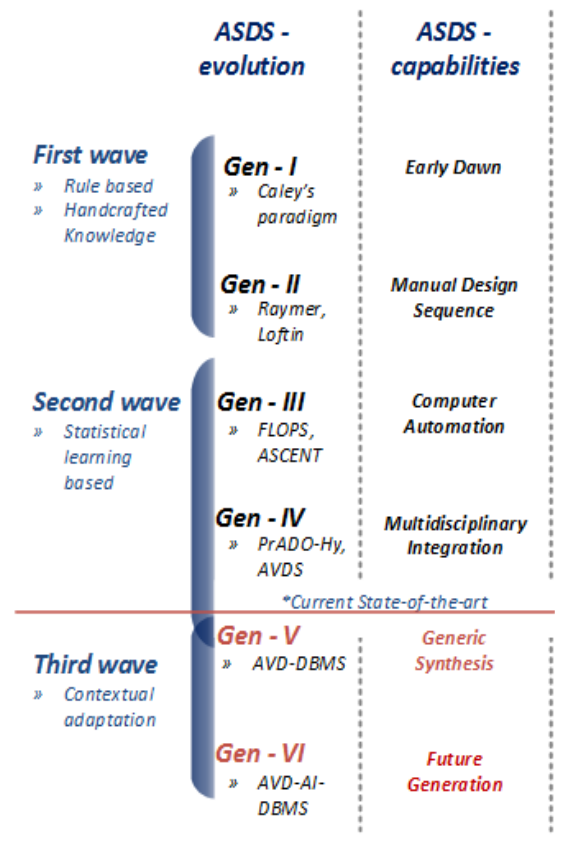

Figure 24 Aerospace Systems Design Synthesis (ASDS) evolution.

\section{References}

[1] F. L. Rowell and J. J. Korte, "Launch vehicle design and optimization methods and priority for the advanced engineering," NASA Langley Research Center, Hampton, VA, 2003.

[2] B. Chudoba, Stability and control of conventional and unconventional aircraft configurations: a generic approach, BoD--Books on Demand, 2001.

[3] X. Huang, "A Prototype Computerized Synthesis Methodology for Generic Space Access Vehicle (SAV) Conceptual Design," Ph.D. Dissertation, The University of Texas at Arlington, Arlington, TX, 2005.

[4] G. Coleman, "Aircraft conceptual design-an adaptable parametric sizing methodology," Ph.D. Dissertation, The University of Texas at Arlington, Arlington, TX, 2010.

[5] L. Gonzalez, "Complex Multidisciplinary System Composition for Aerospace Vehicle Conceptual Design," Ph.D. Dissertation, The University of Texas at Arlington, Alrington, TX, 2016.

[6] A. Omoragbon, "Complex multidisciplinary systems decomposition for aerospace vehicle conceptual design and technology acquisition," Ph.D. Dissertation, The University of Texas at Arlington, Arlington, TX, 2016.

[7] A. Oza, "Integration of a Portfolio-based Approach to Evaluate Aerospace R and D Problem Formulation Into a Parametric Synthesis Tool," Ph.D. Dissertation, The University of Texas at Arlington, Arlington, TX, 2016. 
[8] D. Lovell, "Some experiences with numerical optimisation in aircraft specification and preliminary design studies," in International Congress of the Aerospace Sciences, Munich, 1980.

[9] McDonnell-Douglas, "Hypersonic Research Facilities Study. Volume II Part 2 Phase I Preliminary Studies Flight Vehicle Synthesis," NASA-CR-114324, NASA, 1970.

[10] W. Heinze, "Ein Beitrag Zur Quantitativen Analyse Der Technischen Und Wirtschaftlichen Auslegungsgrenzen Verschiedener Flugzeugkonzepte Fur Den Transport Grosser Nutzlasten," Ph.D. Dissertation, 1994.

[11] E. Torenbeek, "Synthesis of subsonic airplane design, 1982," Delft: Springer.

[12] J. Roskam, Airplane Design: Preliminary Configuration Design and Integration of the Propulsion System, Design Analysis \& Research, 1989, p. 82.

[13] F. Lander and A. Roch, "A summary of the design synthesis process," Society of Aeronautical Weight Engineers Paper, p. 907, 1972.

[14] L. McCullers, "FLOPS: Flight Optimization System," in Proceedings of Recent Experiences in Multidisciplinary Analysis and Optimization, Hampton, VA, 1984.

[15] Microsoft, "Visual Basic: Windows Controls - TreeView Control," Microsoft Developers Networks, 2016. [Online]. Available: https://msdn.microsoft.com/en-us/library/aa443492(v=vs.60).aspx. [Accessed 02 2018].

[16] J. Haley, L. Gonzalez and B. Chudoba, "Generic Hypersonic Vehicle Design Configuration Verification," in AIAA SPACE 2018, Orlando, FL, 2018.

[17] L. Rana, "SPACE ACCESS SYSTEMS DESIGN: Synthesis Methodology Development for Conceptual Design of Future Space Access Systems," Ph.D. Dissertation, The University of Texas at Arlington, Arlington, TX, August, 2017..

[18] L. Rana and B. Chudoba, "Design Evolution and AHP-based Historiography of Lifting Reentry Vehicle Space Programs," in AIAA SPACE 2016, Long Beach, CA, 2016.

[19] P. A. Czysz, C. Bruno and B. Chudoba, Future Spacecraft Propulsion Systems and Integration, Berlin: Springer/Praxis, 2017.

[20] B. Chudoba, "Air-Launched REACH-1 Hypersonic Demonstrator Solution Space Screening: Final Presentation, Air Force Summer Faculty Fellowship Program (SFFP)," High Speed Systems Division, Air Force Research Laboratory, Wright Patterson Air Force Base, 2015.

[21] B. Ruttle, J. Stork and G. Liston, "Generic Hypersonic Vehicles for Conceptual Design Analyses," AFRL, 2012.

[22] L. Rana, T. McCall and B. Chudoba, "Parametric Sizing Boeing X-20 DynaSoar to Gain Program Architectural Understanding of Sierra Nevada Corporation's Dream Chaser (AIAA 2017-5355)," in AIAA SPACE and Astronautics Forum and Exposition, Orlando, FL, 2017.

[23] L. Rana, T. McCall, J. Haley and B. Chudoba, "Parametric Sizing Implementation for Generic Lifting-Body Configuration Executing a Low Earth Orbit Mission (AIAA 2017-5356)," in AIAA SPACE and Astronautics Forum and Exposition, Orlando, FL, 2017.

[24] L. Rana, T. McCall, J. Haley and B. Chudoba, "Conceptual Design Solution Space Identification and Evaluation of Orbital Lifting Reentry Vehicles based on Generic Wing-Body Configuration (AIAA 2017-5127)," in AIAA SPACE and Astronautics Forum and Exposition, Orlando, FL, 2017.

[25] T. McCall, K. Seyed Alavi, L. Rana and B. Chudoba, "Artificial Intelligent Research Assistant for Aerospace Design Synthesis - Solution Logic," in AIAA SPACE 2018, Orlando, FL, 2018. 